Fusion Energy Division

\title{
MULTIPLE ANTENNA IMPLEMENTATION SYSTEM (MAntIS)
}

M. D. Carter

D. B. Batchelor

E. F. Jaeger

DATE PUBLISHED - January 1993

Prepared for the

Office of Fusion Energy

Budget Activity AT 0520210

\author{
Prepared by \\ OAK RIDGE NATIONAL LABORATORY \\ Oak Ridge, Tennessee 37831-6285 \\ managed by \\ MARTIN MARIETTA ENERGY SYSTEMS, INC. \\ for the \\ U.S. DEPARTMENT OF ENERGY \\ under contract DE-AC05-84OR21400
}

MASTER

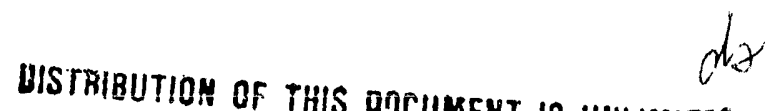




\section{CONTENTS}

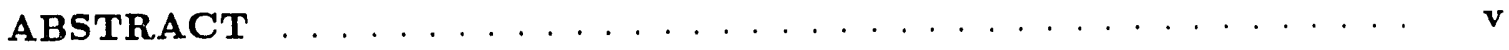

1 MODELLING OF ANTENNAS WITH PLASMA . . . . . . . . . 1

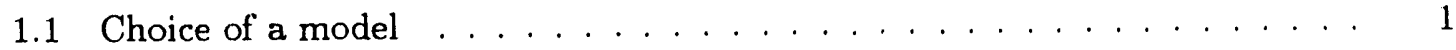

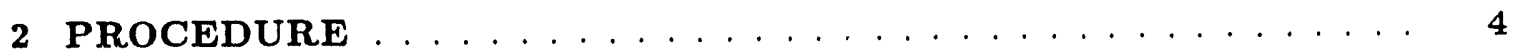

3 REPRESENTATION OF GENERALIZED CURRENT ELEMENTS . 5

4 BOUNDARY CONDITIONS AND MATCHING . . . . . . . . . . T

5 SOLUTION OF PLASMA IMPEDANCE MATRIX . . . . . . . . . 11

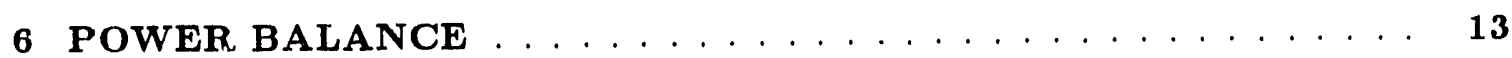

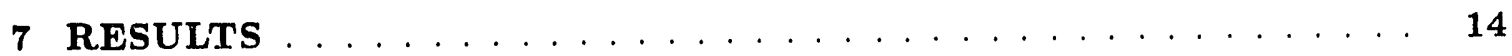

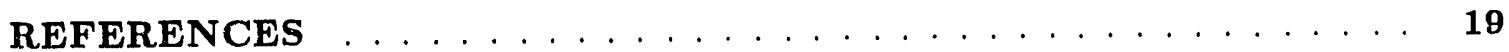




\begin{abstract}
The MAntIS code was developed as an aid to the design of radio frequency (RF) antennas for fusion applications. The code solves for the electromagnetic fields in three dimensions near the antenna structure with a realistic plasma load. Fourier analysis is used in the two dimensions that are tangential to the plasma surface and backwall. The third dimension is handled analytically in a vacuum region with a general impedance match at the plasma-vacuum interface. The impedance tensor is calculated for a slab plasma using the ORION-1D code [JAEGER, E.F., BATCHELOR, D.B., WEITZNER, H., Nuclear Fusion 28 (1988) 53] with all three electric field components included and warm plasma corrections. The code permits the modelling of complicated antenna structures by superposing currents that flow on the surfaces of rectangular parallelepipeds. Specified current elements have feeders that continuously connect the current flowing from the ends of the strap to the feeders. The elements may have an arbitrary orientation with respect to the static magnetic field. Currents are permitted to vary along the length of the current strap and feeders. Parameters that describe this current variation can be adjusted to approximately satisfy boundary conditions on the current elements. The methods used in MAntIS and results for a preliminary loop antenna design are presented.
\end{abstract}




\section{MODILLING OF ANTENNAS WITH PLASMA}

Waves with frequencies in the ion cyclotron (or gyro) range of frequencies (ICRF) have been successfully used to heat plasmas and modify the operation of experimental fusion devices. These radio frequency (RF) systems are attractive for many applications in fusion reactors because they are reliable, effective and relatively inexpensive. Applications for reactors include the driving of steady-state currents, the stabilization of sawteeth, and the auxiliary heating of plasma to ignition.

Crucial components of these RF systems are the antenna and the surrounding structure that directly couple the RF power from a remote transmitter to the fusion-grade plasma. A somewhat simplified example of an RF system to launch fast magnetosonic waves into the plasma, shown in Fig. 1, helps to illustrate the features of a typical antenna. As shown in Fig. 2, feedthroughs at the ends of the current strap are connected to capacitors. These capacitors can be arjusted to help tune the LC circuit to the desired frequency. The current strap in this antenna design is sheltered from the plasma by a row of tubes, referred to as a Faraday shield. Additional protection for the Faraday shield is provided by bumper limiters. These bumper limiters produce a plasma density profile with a very short scale length for radial decay in front of the Faraday shield. The antenna structure is mounted in the shadow of yet another main limiter and/or a divertor that helps isolate the fusion-grade plasma from the structural portions of the fusion device.

The Faraday shield greatly reduces capacitive coupling between the plasma and the parts at high voltage inside the antenna structure, but allows RF magnetic flux to pass through. The antenna is designed so that a maximum in the RF current (and therefore magnetic flux) occurs on the current strap to optimize the power coupling. The RF near fields couple power to the plasma inductively by tunnelling through an evanescent region where the plasma density is too low for wave propagation.

Conflicting requirements must be satisfied when designing an RF antenna system. The antenna must couple a predetermined amount of $\mathrm{RF}$ power to the plasma at a specific frequency. Restrictions may be placed on the Fourier spectrum of the power to achieve a desired result in the plasma. The antenna must fit within a specified region, and it must remain rigid during disruptions. The structure must tolerate heat loads both from the plasma and from Joule dissipation of RF currents The maximum amplitude of the electric fields in the antenna is restricted for two reasons: first, field strengths greater than roughly $2 \mathrm{MV} / \mathrm{m}$ can produce arcing within the antenna structure; second, high values for the electric field near the plasma may give rise to impurities. Ultimately, the plasma loading determines the performance of the antenna.

To simplify the design effort, a computer model is needed for estimating the plasma loading, the maximum electric field, and the Fourier power spectrum. The model should provide this information rapidly so that numerous design alternatives can be considered. The model must include the most important aspects from the plasma physics perspective; however, it should not be unnecessarily complicated.

\subsection{Choice of a model}

Ideally, the model would include at least five effects. It would (1) calculate electromagnetic fields, accounting for structural variations and current flows in all three dimensions, 
(2) solve for the currents in a manner that is self-consistent with all boundary conditions, (3) transiently model all parts of the electrical circuit, including remotely located capacitors and tuning stubs, (4) include fast and slow wave effects in the plasma, and (5) permit finite temperature corrections to the cold plasma approximation. Modelling these effects simultaneously represents a formidable task, so simplification of the problem must be considered.

Commercial three-dimensional (3-D) packages are available to model most of the detail shown in Fig. 1 using finite element or finite difference techniques. However, these packages include only simple dielectric effects, which are not sufficient to determine the plasma loading. These packages are also slow, expensive, and difficult to set up. Thus, for preliminary designs, commercial packages seldom provide information more useful than that available from simpler models that include realistic plasma effects.

Lumped circuit analysis can be used to model a large portion of the RF system. This analysis is valid because most antennas are meant to operate at a fixed frequency for times that are much longer than $1 / \omega$, where $\omega$ is the resonant frequency. Furthermore, the plasma is intended to remain slowly varying during normal $R F$ operation. Thus, feedback circuits can be designed without detailed modelling of the antenna structure [1] if variations in the plasma loading, caused by changes in plasma parameters, are known. Therefore, the explicit modelling of transient effects is usually not necessary in the antenna modelling.

The modelling can be further simplified because the antenna structure typically has a ratio of stored $R F$ field energy to transmitted power that is much larger than $1 / \omega$ (high $Q$ ). Also, lumped circuit transmission-line models and experimental measurements imply that the shape of the current along the current strap does not change dramatically under loading for high $Q$ systems. Thus, a prescribed (not self-consistent) current profile along the current strap, including divergence of the current, is often sufficient to obtain reasonable estimates for plasma loading. Rough estimates for the maximum electric field and power spectrum may also be obtained from a model with a prescribed current profile.

If detailed studies of fields near the Faraday shield are not of interest, then an alternative to explicitly modelling the Faraday shield may be used. One alternative takes advantage of the fact that most plasmas in the edge region of fusion devices are very good conductors in the direction of the static magnetic field. Ignoring any nonlinear changes, the conductivity of the plasma along the static magnetic field produces an effect on the el :tric fields that is very similar to that of a well-designed Faraday shield. Fields found using this alternative can be corrected for the most important effects caused by the shield, such as changes caused by finite thickness in the radial direction.

Fourier analysis in the directions that are tangential to the plasma surface results in tremendous simplification of the interface between vacuum and plasma. Plasma loading results and information about the power spectrum can be obtained rapidly without reconstructing the fields in real space. General current descriptions can be constructed and one-dimensional solutions in the radial direction can be found rapidly by analytic or numerical techniques.

Estimating the maximum electric fields near the current strap requires distributing charge along the strap by permitting a non-zero divergence of the current density. Elcctrostatic fields resulting from this buildup of charge can be very large, especially when the current strap length becomes an appreciable fraction of the free space wavelength. The variation of the current along the strap from transmission-line analysis is frequently called the finite phase velocity, $v_{\phi}$, where $v_{\phi}=\lambda \omega / 2 \pi$ and $\lambda_{s}$ is the effective wavelength describing 
the current variation along the strap. Capacitive effects from the Faraday shield can cause $v_{\phi}$ to be significantly less than the speed of light in a vacuum. For example, a current strap with a length of $\lambda / 2$ would have a null in the current at some point along the strap and therefore an extremum in the charge. This charge causes a radial electric field between the current strap and the Faraday shield. This radial electric field must be considered in the model if it is large enough to cause arcing or produce impurities. To prevent an artificial divergence of the current at the ends of the current strap, feeders that carry away residual currents are needed.

With these considerations, Fourier analysis in two directions with coupling to a slab plasma was chosen as the method for modelling in MAntIS. The cross-section of the antenna structure shown in Fig. 2 illustrates the basic components modelled in MAntIS. The ORION$1 \mathrm{D}$ code [2] was chosen to calculate the impedance match at the plasma-vacuum interface in the same manner as that used by Brambilla [3]. Current strap representations allow variation of the current along the current strap and feeders. Continuity is maintained at the connections between the current strap and the feeders. A general variational principle [4] was not used, but minimization techniques can be used to approximately satisfy boundary conditions on the current strap. Multiple current elements with standing wave patterns on the feeder elements were implemented to model RF systems based on the folded waveguide concept $[5,6]$. The Faraday shield is not explicitly modelled in MAntIS; however, corrections can be made for a Faraday shield aligned with the static magnetic field, as demonstrated in Section 7 for a current strap with length of roughly $\lambda / 2$.

The remainder of the paper is organized as follows. Section 2 describes the equations that are solved by MAntIS and the Fourier analysis used. Section 3 describes the methods used to represent basic current elements (which include feeders). Section 4 describes the boundary and matching conditions used in the vacuum region and gives the analytic field solutions in Fourier space. Section 5 briefly describes the calculation of the plasma impedance matrix using the ORION-1D code. Section 6 describes diagnostics that are used to ensure that the appropriate conservation relations are satisfied. Section 7 gives the results of a singleelement model for a simple loop antenna design using finite phase velocity along the current strap to obtain electric field limitations on plasma coupling. 


\section{PROCEDURE}

Maxwell's equations in a vacuum region for fields with a single frequency are completely described by

$$
\nabla^{2} \vec{E}+\frac{\omega^{2}}{c^{2}} \vec{E}-\nabla(\nabla \cdot \vec{E})=-i \omega \mu_{0} \vec{J}
$$

where $\vec{E}$ and $\vec{J}$ are complex quantities referring to the electric field and current respectively, omitting the $\exp (-i \omega t)$ dependence. Following Figs 1 and 2 and as $t:$ scussed in Section 1, the region near the antenna is modelled as a periodic slab. The slab represents a torus with minor radius $a$ and major radius $R$. Differences between $a_{p}$ and $a_{s}$, as shown in Fig. 1, are typically small. The expansion in the slab model typically uses a value of $a \approx a_{p}$ and $R$ is taken at the magnetic axis.

Using a Cartesian coordinate system with $y$ in the poloidal direction and $z$ in the tornidal direction, we expand the field and current components using a complete basis over the range $-\pi a \leq y \leq \pi a$ and $-\pi R \leq z \leq \pi R$, e.g.

$$
\vec{J}(x, y, z)=\sum_{m, n} \vec{J}^{m, n}(x) \exp \{i[(m y / a)+(n z / R)]\}
$$

with

$$
\begin{aligned}
\vec{J}^{m, n}(x)= & \frac{1}{4 \pi^{2} a R} \int_{-\pi a}^{\pi a} \int_{-\pi R}^{\pi R} \vec{J}(x, y, z) \\
& \quad \times \exp \{-i[(m y / a)+(n z / R)]\} d z d y
\end{aligned}
$$

where $m$ and $n$ are integers ranging from $-\infty$ to $\infty$. (The explicit $x$ dependence will be dropped in most instances, but is retained for emphasis in some equations.) Charge conservation and Maxwell's equations give $\nabla \cdot \vec{J}=i \omega \rho=i \omega \nabla \cdot \vec{E} /\left(\mu_{0} c^{2}\right)$. Using this relationship and applying the Fourier analysis implied by Eq. (2) to Eq. (1), we find that the Fourier components satisfy

$$
\begin{aligned}
\frac{d^{2} E_{y}^{m, n}}{d x^{2}} & +k_{\perp}^{2 m, n} E_{y}^{m, n}= \\
& -i \omega \mu_{0}\left[J_{y}^{m, n}+\frac{i c^{2}}{\omega^{2}} \frac{m}{a}(\nabla \cdot \vec{J})^{m, n}\right] \\
\frac{d^{2} E_{z}^{m, n}}{d x^{2}} & +k_{\perp}^{2 m, n} E_{z}^{m, n}= \\
& -i \omega \mu_{0}\left[J_{z}^{m, n}+\frac{i c^{2}}{\omega^{2}} \frac{n}{R}(\nabla \cdot \vec{J})^{m, n}\right]
\end{aligned}
$$

where $\vec{J}$ is treated as a source term. The $E_{x}^{m, n}$ component can be determined from the solutions to Eqs (4a) and (4b) by Fourier analysis of the $x$-component of Eq. (1), giving

$$
\begin{aligned}
k_{\perp}^{2 m, n} E_{x}^{m, n}= & -i \omega \mu_{0} J_{x}^{m, n}+\frac{i m}{a} \frac{d E_{y}^{m, n}}{d x} \\
& +\frac{i n}{R} \frac{d E_{z}^{m, n}}{d x}
\end{aligned}
$$

where $k_{\perp}^{2 m, n}=\omega^{2} / c^{2}-m^{2} / a^{2}-n^{2} / R^{2}$. The notation $(\nabla \cdot \vec{J})^{m, n}$ refers to the Fourier components of the time derivative of the charge density, $i \omega \rho^{m, n}(x)$. 


\section{REPRESENTATION OF GENERALIZED CURRENT ELEMENTS}

In choosing the basic element for modelling antenna structures in MAntIS, one consid. eration was to ensure that general modelling of complicated antenna structures could be readily performed by superposition. Another consideration was to choose the element in a way that allowed the current at the ends of the strap to be continuous with the current on the connecting ends of the feeders. Enforcing this continuity by analytic means is important so that artificial singularities need not be cancelled numerically.

The basic elements for representing the geometry of an antenna in MAntIS are rectangular parallelepipeds. The RF currents flow on the surfaces of these parallelepipeds. Complicated structures can be modelled using an arbitrary number of basic elements. These elements are represented by introducing a geometry function, $f(x, y, z)$, that has a value of one inside the parallelepiped and zero outside. As shown in Fig. 3, we introduce a Cartesian coordinate system, $\left(x^{\prime}, y^{\prime}, z^{\prime}\right)$, that is rotated around the $x$-axis by an angle $\theta$ from the $z$-axis. The transformation to this coordinate system is $x=x^{\prime}, y=y_{c}+y^{\prime} \cos (\theta)+z^{\prime} \sin (\theta)$ and $z=z_{c}-y^{\prime} \sin (\theta)+z^{\prime} \cos (\theta)$. Heaviside step functions, $\Theta$, describe $f$, giving

$$
\begin{aligned}
& f\left(x^{\prime}, y^{\prime}, z^{\prime}\right)=\Theta\left(x^{\prime}-d\right)\left[\Theta\left(y^{\prime}+\frac{\alpha}{2}\right)\right. \\
& \left.-\Theta\left(y^{\prime}-\frac{\alpha}{2}\right)\right]\left[\Theta\left(z^{\prime}+\frac{\beta}{2}\right)-\Theta\left(z^{\prime}-\frac{\beta}{2}\right)\right]
\end{aligned}
$$

The feeders are included in the basic element, and are arbitrarily chosen to be at $z^{\prime}= \pm \beta / 2$ without loss of generality. We simplify the model by constraining the currents to flow in the $z^{\prime}$-direction on the current strap at $x=d$ and in the $x$-direction on the feeders.

The currents flowing on the strap at $x=d$ are described by a surface current function, $K(y, z)$, that is continuous and defined throughout the periodic domain. Thus, the current density on this element is modelled by

$$
\vec{J}=K(y, z) \frac{\partial f(x, y, z)}{\partial x}(\cos \theta \hat{z}+\sin \theta \hat{y})+J_{x} \hat{x}
$$

The current density on the feeders is chosen to be

$$
J_{\mathfrak{x}}=-K(y, z)\left[\cos \theta \frac{\partial f}{\partial z}+\sin \theta \frac{\partial f}{\partial y}\right] j_{\mathfrak{x}}(x)
$$

where the complex function, $j_{x}(x)$, describes the variation of current along the feeders. Constraining the value of $j_{x}(x=d)=1$ ensures continuity of the currents flowing from the main strap onto the feeders at $z^{\prime}= \pm \beta / 2$. A general expansion for $j_{x}$ gives

$$
j_{x}(x)=\sum_{p=-\infty}^{\infty} j_{p} \exp \left[i p \pi(x-d) / a_{w}\right]
$$

with

$$
\sum_{p=-\infty}^{\infty} j_{p}=1
$$


and

$$
j_{p}=\frac{1}{2 a_{w}} \int_{d-a_{w}}^{i\left\llcorner a_{w}\right.} j_{x}(x) \exp \left[-i p \pi(x-d) / a_{w}\right] d x
$$

The step behaviour in the geometry function, $f$, restricts the use of $j_{x}$ to the range from $x=d$ to $x=d+a_{w}$.

The Fourier analysis for the geometry function is carried out by applying the example of Eq. (3) to Eq. (5). The integration is carried out in the $\left(x^{\prime}, y^{\prime}, z^{\prime}\right)$ coordinate system, where the step functions can be used to limit the range of integration. The result is

$$
\begin{aligned}
f^{m, n}(x)= & \exp \left\{-i\left[\left(m y_{c} / a\right)+\left(n z_{c} / R\right)\right]\right\} \frac{\Theta(x-d)}{\pi^{2} a R} \\
& \times \frac{\sin \{\alpha[(m / a) \cos \theta-(n / R) \sin \theta] / 2\}}{[(m / a) \cos \theta-(n / R) \sin \theta]} \\
& \times \frac{\sin \{\beta[(m / a) \sin \theta+(n / R) \cos \theta] / 2\}}{[(m / a) \sin \theta+(n / R) \cos \theta]} \\
f^{0,0}(x)= & \frac{\alpha \beta}{4 \pi^{2} a R} \Theta(x-d)
\end{aligned}
$$

Fourier components of the currents are obtained by using convolutions of $K$ with $f . K$ can be used to represent spatial variations in the driven RF current that arise from finite phase velocity, e.g. $K\left(y^{\prime}, z\right)=\cos \left(\omega y^{\prime} / v_{\phi}\right)$. Using orthogonality of the basic f.nctions, we find the Fourier components of $\vec{J}$ from Eq. (6),

$$
\begin{aligned}
\vec{J}^{m, n}= & \sum_{m^{\prime}, n^{\prime}} K^{m^{\prime}, n^{\prime}} \frac{d f}{d x} \\
& \times(\cos \theta \hat{z}+\sin \theta \hat{y})+J_{x}^{m, n} \hat{x}
\end{aligned}
$$

When a finite number of Fourier modes is used, twice as many modes must be retained for the $f$ function as for the $K$ function to produce the correct $\vec{J}^{m, n}$ coefficients.

In Fourier space, the feeder currents are

$$
\begin{aligned}
J_{x}^{m, n} \equiv & \mathcal{J}_{x}^{m, n} j_{x}(x)=-i \sum_{m^{\prime}, n^{\prime}} f^{m-m^{\prime}, n-n^{\prime}} K^{m^{\prime}, n^{\prime}} \\
& \times\left(\frac{m-m^{\prime}}{a} \sin \theta+\frac{n-n^{\prime}}{R} \cos \theta\right) j_{x}(x)
\end{aligned}
$$

Feeder currents in the $x$ direction require particular solutions for Eqs (4a) and (4b), if $d j_{x}(x) / d x$ is not zero. These solutions are obtained analytically by using Eqs (7) for $j_{x}$ in Section 4 . 


\section{BOUNDARY CONDITIONS AND MATCHING}

For each basic current element, the current-free region between the plasma-vacuum interface at $x=0$ and the current strap at $x=d$ is denoted Region II (see Fig. 3). The region between the strap at $x=d$ and the backwall at $x=d+a_{w}$ is denoted Region I. A specified electric field is applied for the boundary condition at the backwall. For example, $E_{y}^{m, n}\left(x=d+a_{w}\right)=0$ and $E_{z}^{m, n}\left(x=d+a_{w}\right)=0$ is the boundary condition for a perfectly conducting backwall.

At the interface between Regions I and II, the delta function, $\delta(x-d)$, arising from $\partial f / \partial x$ is treated analytically. Thus, Eqs (4a) and (4b) are integrated across the infinitesimal boundary at $x=d$ while maintaining continuity for both the $E_{y}^{m, n}$ and $E_{z}^{m, n}$ components. ( $\dot{E}_{x}^{m, n}$ can be discontinuous at $x=d$.) In Region !, the delta functions arising from $\partial f / \partial y$ and $\partial f / \partial z$ are true discontinuities only in the limit that an infinite number of Fourier modes is considered. Thus, the scale length of resolution for the feeders and the ends of the main strap depends upon the largest Fourier harmonic retained in the calculation.

With Eqs (7a) and (9b), the solutions to Eqs (4a) and (4b) in Region I are

$$
\begin{aligned}
E_{y \mathrm{I}}^{m, n}=A_{\mathrm{I}}^{m, n} & \exp \left[i k_{\perp}^{m, n}\left(x-d-a_{w}\right)\right] \\
& +B_{\mathrm{I}}^{m, n} \exp \left[-i k_{\perp}^{m, n}\left(x-d-a_{w}\right)\right] \\
& +\mathrm{P}_{y}^{m, n}(x) \\
E_{z \mathrm{I}}^{m, n}=C_{\mathrm{I}}^{m, n} & \exp \left[i k_{\perp}^{m, n}\left(x-d-a_{w}\right)\right] \\
& +D_{\mathrm{I}}^{m, n} \exp \left[-i k_{\perp}^{m, n}\left(x-d-a_{w}\right)\right] \\
& +\mathrm{P}_{z}^{m, n}(x)
\end{aligned}
$$

where

$$
\begin{aligned}
\mathrm{P}_{y}^{m, n}(x)= & \frac{i \mu_{0} \pi c^{2}}{\omega a_{w}} \frac{m}{a} \mathcal{J}_{x}^{m, n} \\
& \times \sum_{p=-\infty}^{\infty} \frac{p j_{p} \exp \left[i p \pi(x-d) / a_{w}\right]}{k_{\perp}^{2 m, n}-p^{2} \pi^{2} / a_{w}^{2}}
\end{aligned}
$$

and

$$
\begin{aligned}
\mathrm{P}_{z}^{m, n}(x)= & \frac{i \mu_{0} \pi c^{2}}{\omega a_{w}} \frac{n}{R} \mathcal{J}_{x}^{m, n} \\
& \times \sum_{p=-\infty}^{\infty} \frac{p j_{p} \exp \left[i p \pi(x-d) / a_{w}\right]}{k_{\perp}^{2 m, n}-p^{2} \pi^{2} / a_{w}^{2}}
\end{aligned}
$$

Using the notation, $E_{y \mathrm{I}}^{m, n}\left(x=d+a_{w}\right)$, to represent the specified field at the boundary, $x=d+a_{w}$, gives

$$
\begin{aligned}
& B_{\mathrm{I}}^{m, n}=E_{y \mathrm{I}}^{m, n}\left(x=d+a_{w}\right)-A_{\mathrm{I}}^{m, n} \\
& \quad-\frac{i \mu_{0} \pi c^{2}}{\omega a_{w}} \frac{m}{a} J_{x}^{m, n} \sum_{p=-\infty}^{\infty} \frac{p(-1)^{p} j_{p}}{k_{\perp}^{2 m, n}-p^{2} \pi^{2} / a_{w}^{2}}
\end{aligned}
$$


and

$$
\begin{aligned}
& D_{\mathrm{I}}^{m, n}=E_{z \mathrm{I}}^{m, n}\left(x=d+a_{w}\right)-C_{\mathrm{I}}^{m, n} \\
& \quad-\frac{i \mu_{0} \pi c^{2}}{\omega a_{w}} \frac{n}{R} \mathcal{J}_{x}^{m, n} \sum_{p=-\infty}^{\infty} \frac{p(-1)^{p} j_{p}}{k_{\perp}^{2 m, n}-p^{2} \pi^{2} / a_{w}^{2}}
\end{aligned}
$$

In Region II, the solutions to Eqs (4a) and (4b) are

$$
\begin{aligned}
& E_{y I I}^{m, n}=A_{\mathrm{II}}^{m, n} \exp \left(i k_{\perp}^{m, n} x\right)+B_{\mathrm{II}}^{m, n} \exp \left(-i k_{\perp}^{m, n} x\right) \\
& E_{z \mathrm{II}}^{m, n}=C_{\mathrm{II}}^{m, n} \exp \left(i k_{\perp}^{m, n} x\right)+D_{\mathrm{II}}^{m, n} \exp \left(-i k_{\perp}^{m, n} x\right)
\end{aligned}
$$

Integration of Eqs (4a) and (4b) across the $x=d$ location yields the jump condition for the derivatives at $x=d$ :

$$
\left.\frac{d E_{y \mathrm{I}}^{m, n}}{d x}\right|_{x=d}-\left.\frac{d E_{y \mathrm{II}}^{m, n}}{d x}\right|_{x=d}=-i \omega \mu_{0} \cdot K_{y}^{m, n}
$$

where

$$
\begin{aligned}
\mathcal{K}_{y}^{m, n} \equiv & \sum_{m^{\prime}, n^{\prime}} f^{m-m^{\prime}, n-n^{\prime}} K^{m^{\prime}, n^{\prime}} \\
& \times\left[\left(1-\frac{c^{2}}{\omega^{2}} \frac{m m^{\prime}}{a^{2}}\right) \sin \theta-\frac{c^{2}}{\omega^{2}} \frac{m n^{\prime}}{a R} \cos \theta\right]
\end{aligned}
$$

and

$$
\left.\frac{d E_{z I}^{m, n}}{d x}\right|_{x=d}-\left.\frac{d E_{z I I}^{m, n}}{d x}\right|_{x=d}=-i \omega \mu_{0} \mathcal{K}_{z}^{m, n}
$$

where

$$
\begin{aligned}
\mathcal{K}_{z}^{m, n} \equiv & \sum_{m^{\prime}, n^{\prime}} f^{m-m^{\prime}, n-n^{\prime}} K^{m^{\prime}, n^{\prime}} \\
& \times\left[\left(1-\frac{c^{2}}{\omega^{2}} \frac{n n^{\prime}}{R^{2}}\right) \cos \theta-\frac{c^{2}}{\omega^{2}} \frac{n m^{\prime}}{a R} \sin \theta\right]
\end{aligned}
$$

The surface terms, $\mathcal{K}_{y}^{m, n}$ and $\mathcal{K}_{z}^{m, n}$, include both current and charge sources for the fields.

Differentiating Eqs (10) and (11) for use in Eq. (12), we find that the jump condition at $x=d$ becomes

$$
\begin{aligned}
& i k_{\perp}^{m, n}\left[A_{\mathrm{II}}^{m, n} \exp \left(i k_{\perp}^{m, n} d\right)-B_{\mathrm{II}}^{m, n} \exp \left(-i k_{\perp}^{m, n} d\right)\right]= \\
& \quad i k_{\perp}^{m, n}\left[A_{\mathrm{I}}^{m, n} \exp \left(-i k_{\perp}^{m, n} a_{w}\right)-B_{\mathrm{I}}^{m, n} \exp \left(i k_{\perp}^{m, n} a_{w}\right)\right] \\
& \quad+i \omega \mu_{0} \mathcal{K}_{y}^{m, n}+\left.\frac{d \mathrm{P}_{y}^{m, n}}{d x}\right|_{x=d} \\
& \quad i k_{\perp}^{m, n}\left[C_{\mathrm{II}}^{m, n} \exp \left(i k_{\perp}^{m, n} d\right)-D_{\mathrm{II}}^{m, n} \exp \left(-i k_{\perp}^{m, n} d\right)\right]= \\
& \quad i k_{\perp}^{m, n}\left[C_{\mathrm{I}}^{m, n} \exp \left(-i k_{\perp}^{m, n} a_{w}\right)-D_{\mathrm{I}}^{m, n} \exp \left(i k_{\perp}^{m, n} a_{w}\right)\right] \\
& \quad+i \omega \mu_{0} K_{z}^{m, n}+\left.\frac{d \mathrm{P}_{z}^{m, n}}{d x}\right|_{x=d}
\end{aligned}
$$


and the match condition at $x=d$ is obtained using Eqs (10) and (11) to give

$$
\begin{aligned}
A_{\mathrm{II}}^{m, n} \exp \left(i k_{\perp}^{m, n} d\right) & +B_{\mathrm{II}}^{m, n} \exp \left(-i k_{\perp}^{m, n} d\right)=A_{\mathrm{I}}^{m, n} \exp \left(-i k_{\perp}^{m, n} a_{w}\right) \\
& +B_{\mathrm{I}}^{m, n} \exp \left(i k_{\perp}^{m, n} a_{w}\right)+\mathrm{P}_{y}^{m, n}(x=d)
\end{aligned}
$$

and

$$
\begin{aligned}
C_{\mathrm{II}}^{m, n} \exp \left(i k_{\perp}^{m, n} d\right) & +D_{\mathrm{II}}^{m, n} \exp \left(-i k_{\perp}^{m, n} d\right)=C_{\mathrm{I}}^{m, n} \exp \left(-i k_{\perp}^{m, n} a_{w}\right) \\
& +D_{\mathrm{I}}^{m, n} \exp \left(i k_{\perp}^{m, n} a_{w}\right)+\mathrm{P}_{z}^{m, n}(x=d)
\end{aligned}
$$

Using Eq. (13), we can write the Region II coefficients in terms of $A_{1}^{m, n}$ and $C_{I}^{m, n}$ so that Eq. (11) becomes

$$
\begin{aligned}
& E_{y \mathrm{II}}^{m, n}=A_{\mathrm{I}}^{m, n}\left\{\exp \left[i k_{\perp}^{m, n}\left(x-a_{w}-d\right)\right]-\exp \left[-i k_{\perp}^{m, n}\left(x-a_{w}-d\right)\right]\right\} \\
& +\frac{\omega \mu_{0}}{2 k_{\perp}^{m, n}} \mathcal{K}_{y}^{m, n}\left\{\exp \left[i k_{\perp}^{m, n}(x-d)\right]-\exp \left[-i k_{\perp}^{m, n}(x-d)\right]\right\} \\
& +E_{y}^{m, n}\left(x=d+a_{w}\right) \exp \left[-i k_{\perp}^{m, n}\left(x-a_{w}-d\right)\right] \\
& +\frac{i \mu_{0} \pi c^{2}}{\omega a_{w}} \frac{m}{a} \mathcal{J}_{x}^{m, n} \sum_{p=-\infty}^{\infty} \frac{p j_{p}}{k_{\perp}^{2 m, n}-p^{2} \pi^{2} / a_{w}^{2}} \\
& \times\left\{\frac{1}{2}\left(\frac{p \pi}{k_{\perp}^{m, n} a_{w}}+1\right) \exp \left[i k_{\perp}^{m, n}(x-d)\right]\right. \\
& -\frac{1}{2}\left(\frac{p \pi}{k_{\perp}^{m, n} a_{w}}-1\right) \exp \left[-i k_{\perp}^{m, n}(x-d)\right] \\
& \left.-(-1)^{p} \exp \left[-i k_{\perp}^{m, n}\left(x-d-a_{w}\right)\right]\right\} \\
& E_{z I I}^{m, n}=C_{I}^{m, n}\left\{\exp \left[i k_{\perp}^{m, n}\left(x-a_{w}-d\right)\right]-\exp \left[-i k_{\perp}^{m, n}\left(x-a_{w}-d\right)\right]\right\} \\
& +\frac{\omega \mu_{0}}{2 k_{\perp}^{m, n}} \mathcal{K}_{z}^{m, n}\left\{\exp \left[i k_{\perp}^{m, n}(x-d)\right]-\exp \left[-i k_{\perp}^{m, n}(x-d)\right]\right\} \\
& +E_{z}^{m, n}\left(x=d+a_{w}\right) \exp \left[-i k_{\perp}^{m, n}\left(x-a_{w}-d\right)\right] \\
& +\frac{i \mu_{0} \pi c^{2}}{\omega a_{w}} \frac{n}{R} \mathcal{J}_{x}^{m, n} \sum_{p=-\infty}^{\infty} \frac{p j_{p}}{k_{\perp}^{2 m, n}-p^{2} \pi^{2} / a_{w}^{2}} \\
& \times\left\{\frac{1}{2}\left(\frac{p \pi}{k_{\perp}^{m, n} a_{w}}+1\right) \exp \left[i k_{\perp}^{m, n}(x-d)\right]\right. \\
& -\frac{1}{2}\left(\frac{p \pi}{k_{\perp}^{m, n} a_{w}}-1\right) \exp \left[-i k_{\perp}^{m, n}(x-d)\right] \\
& \left.-(-1)^{p} \exp \left[-i k_{\perp}^{m, n}\left(x-d-a_{w}\right)\right]\right\}
\end{aligned}
$$

The last two equations for the Region I coefficients are obtained by considering the match between the vacuurn region (Region II) and the plasma. The match is taken to be 
in the vacuum just outside the plasma surface, so that if no source currents pass through the interface between the vacuum region and the plasma surface $(\vec{J}(x=0)=0)$, then continuity of the magnetic field implies that the matching condition can take the form of an impedance matrix [3]. The impedance match at $x=0$ is written with the assumption that toroidal and poloidal modes are not coupled (periodic slab approximation), giving

$$
\begin{aligned}
E_{y}^{m, n}(x=0)=\frac{1}{\mu_{0}}[ & Z_{11}^{m, n} B_{y}^{m, n} \\
& \left.+\mathcal{Z}_{12}^{m, n} B_{z}^{m, n}\right]_{x=0} \\
E_{z}^{m, n}(x=0)=\frac{1}{\mu_{0}}[ & Z_{21}^{m, n} B_{y}^{m, n} \\
& \left.+\mathcal{Z}_{22}^{m, n} B_{z}^{m, n}\right]_{x=0}
\end{aligned}
$$

where the $\mathcal{Z}_{i j}^{m, n}$ coefficients of the impedance matrix are calculated as described in Section 5 and the notation $[\ldots]_{x=0}$ indicates that all field components inside the brackets are to be evaluated at $x=0$. The tangential components of $\nabla \times \vec{E}=i \omega \vec{B}$ in Fourier space give

$$
\begin{aligned}
& {\left[i \omega B_{y}^{m, n}=\frac{i n}{R} E_{x}^{m, n}-\frac{d E_{z}^{m, n}}{d x}\right]_{x=0}} \\
& {\left[i \omega B_{z}^{m, n}=\frac{d E_{y}^{m, n}}{d x}-\frac{i m}{a} E_{x}^{m, n}\right]_{x=0}}
\end{aligned}
$$

Thus, if the impedance matrix is known (see Section 5), then Eq. (14) can be used to calculate $d E_{y}^{m, n} / d x$ and $d E_{z}^{m, n} / d x$ in Region II at $x=0$. These derivatives at $x=0$ are then used in Eq. (4c) to eliminate $E_{x}^{m, n}(x=0)$ in Eqs (15c) and (15d). The resulting two equations relate the two unknown Region I coefficients, $A_{\mathrm{I}}^{m, n}$ and $C_{\mathrm{I}}^{m, n}$, by using Eq. (14) to evaluate $E_{y}^{m, n}$ and $E_{z}^{m, n}$ at $x=0$.

Specific solutions for $A_{\mathrm{I}}^{m, n}$ and $C_{\mathrm{I}}^{m, n}$ for wwo feeder options have been tested in MAntIS and are given in the Appendix. The first option includes currents that are constant and continuous along the feeder. The second feeder option permits both standing wave and travelling wave currents with wavelength $2 a_{w}$ to flow on the feeder elements. This option has been used primarily for folded waveguide modelling $[i, 6]$. 


\section{SOLUTION OF PLASMA IMPEDANCE MATRIX}

The calculation of the impedance matrix for the plasma-vacuum interface is obtained by using the ORION-1D code [2]. ORION-1D models a slab plasma using Fourier analysis in the direction of the static magnetic field and in the direction orthogonal to both pressure gradients and the static magnetic field. Periodicity conditions for the directions that are Fourier analysed are chosen to represent the poloidal and toroidal directions shown in Fig. 1. The equations solved are the three components of

$$
-\nabla \times \nabla \times \vec{E}+\frac{\omega^{2}}{c^{2}} \vec{E}+i \omega \mu_{0} \sum_{\mathbf{s}} \vec{J}_{\mathbf{s}}=\vec{S}
$$

where the summation is over the plasma species s and $\vec{S}$ is an external source term. The plasma current density terms, $\vec{J}_{\mathbf{s}}$, are calculated using second-order finite temperature corrections to the warm plasma dielectric tensor. A sixth-order finite difference technique is used to solve Eq. (16) in the direction of the plasma density and temperature gradients. The numerical implementation used to calculate the impedance matrix is described below.

Faraday's law, $\nabla \times \vec{E}=i \omega \vec{B}$, applies at all finite difference nodes regardless of the presence of plasma currents. With a conservative finite difference scheme, the numerical representation of Faraday's law is

$$
\begin{gathered}
i k_{y} E_{z N}^{m, n}-i k_{z} E_{y N}^{m, n}=i \omega B_{x N}^{m, n} \\
i k_{z} E_{x N-1}^{m, n}-\left(E_{z N}^{m, n}-E_{z N-1}^{m, n}\right) / \Delta=i \omega B_{y N-1}^{m, n} \\
\left(E_{y N}^{m, n}-E_{y N-1}^{m, n}\right) / \Delta-i k_{y} E_{x N-1}^{m, n}=i \omega B_{z N-1}^{m, n}
\end{gathered}
$$

where $k_{y}=m / a$ and $k_{z}=n / R$ for periodic boundaries in the $y$ - and $z$-directions. In Eq. (17), $N$ refers to the numerical nodes just on the vacuum side of the plasma-vacuum boundary and $\Delta$ is the discrete step size in the $x$-direction. Field values at these last two locations (separated by $\Delta / 2$ ) represent the numerical resolution of the plasma-vacuum boundary. The correspondences betwreen field values and node location are shown in Fig. 4 and are such that the numerical implementation of Stokes' theorem and the divergence theorem are satisfied. The remaining Maxwell's equation is written for this node as

$$
\begin{gathered}
i k_{y} B_{z N}^{m, n}-i k_{z} B_{y N}^{m, n}=\mu_{0} J_{x N}^{m, n}-\frac{i \omega}{c^{2}} E_{x N}^{m, n} \\
i k_{z} B_{x N}^{m, n}-\left(B_{z N}^{m, n}-B_{z N-1}^{m, n}\right) / \Delta \\
=\mu_{0} J_{y N}^{m, n}-\frac{i \omega}{c^{2}} E_{y N}^{m, n} \\
\left(B_{y N}^{m, n}-B_{y N-1}^{m, n}\right) / \Delta-i k_{y} B_{x N}^{m, n} \\
=\mu_{0} J_{z N}^{m, n}-\frac{i \omega}{c^{2}} E_{z N}^{m, n}
\end{gathered}
$$

Using Eq. (17) to eliminate $B_{x N}^{m, n}, B_{y N-1}^{m, n}$, and $B_{z N-1}^{m, n}$ in Eq. (18) and considering $B_{y N}^{m, n}$ and $B_{z N}^{m, n}$ to be source terms, we obtain the numerical boundary equations at the impedance 
match location $(x=0)$ :

$$
\begin{gathered}
E_{x N}^{m, n}=\frac{-i c^{2}}{\omega}\left(\mu_{0} J_{x N}^{m, n}-i k_{y} B_{z N}^{m, n}\right. \\
\left.+i k_{y} B_{y N}^{m, n}\right) \\
\frac{i c^{2}}{\omega^{2}} \frac{k_{y}}{\Delta} E_{x N-1}^{m, n}+\frac{c^{2}}{\omega^{2}} \frac{E_{y N-1}^{m, n}}{\Delta^{2}}+\frac{c^{2}}{\omega^{2}} k_{y} k_{z} E_{z N}^{m, n} \\
+\left[1-\frac{c^{2}}{\omega^{2}}\left(k_{z}^{2}+1 / \Delta^{2}\right)\right] E_{y N}^{m, n} \\
=\frac{-i c^{2}}{\omega} \mu_{0}\left(J_{y N}^{m, n}+\frac{1}{\mu_{0} \Delta} B_{z N}^{m, n}\right) \\
\frac{i c^{2}}{\omega^{2}} \frac{k_{z}}{\Delta} E_{x N-1}^{m, n}+\frac{c^{2}}{\omega^{2}} k_{y} k_{z} E_{y N}^{m, n}+\frac{c^{2}}{\omega^{2}} \frac{E_{z N-1}^{m, n}}{\Delta^{2}} \\
+\left[1-\frac{c^{2}}{\omega^{2}}\left(1 / \Delta^{2}+k_{y}^{2}\right)\right] E_{z N}^{m, n} \\
=\frac{-i c^{2}}{\omega} \mu_{0}\left(J_{z N}^{m, n}-\frac{1}{\mu_{0} \Delta} B_{y N}^{m, n}\right)
\end{gathered}
$$

Because the impedance match is in a vacuum region, and it is assumed that no antenna source currents penetrate the plasma surface, all components at the $N$ th nodes of $\vec{J}^{m, n}$ are zero (see Fig. 4). The impedance tensor, $\overrightarrow{\overrightarrow{\mathcal{Z}}}$, described in Eqs (15a) and (15b) is systematically calculated by setting $B_{y N}^{m, n}=\mu_{0}$ and $B_{z N}^{m, n}=0$ to obtain $\mathcal{Z}_{11}^{m, n}$ and $\mathcal{Z}_{21}^{m, n}$ from the $E_{y N}^{m, n}$ and $E_{z N}^{m, n}$ solutions provided by ORION-1D. The $\boldsymbol{Z}_{12}^{m, n}$ and $\boldsymbol{Z}_{22}^{m, n}$ coefficients are similarly found by setting $B_{z N}^{m, n}=\mu_{0}$ and $B_{y N}^{m, n}=0$. The impedance tensor is calculated for a desired range of $m$ and $n$ values and plasma parameters and can be used for many subsequent MAntIS runs.

At the wall opposite the plasma impedance matching location, ORION-1D makes a transition from warm to cold plasma and then applies a conducting wall boundary condition. An outgoing boundary condition is presently simulated by using an artificial absorber [2]. 


\section{POWER BALANCE}

Solutions obtained by MAntIS were checked by ensuring that

$$
-\frac{1}{2} \operatorname{Re} \int_{v} \vec{E} \cdot \vec{J}^{*} d v=\frac{1}{2 \mu_{0}} \operatorname{Re} \int_{s}\left(\vec{E} \times \vec{B}^{*}\right)_{x} d s
$$

where the asterisk $*$ indicates the complex conjugate, $v$ denotes the volume integral, $s$ denotes the plasma surface integral and the subscript $x$ denotes the component perpendicular to the plasma surface. Wave energy conservation was computed by three separate means to ensure that the coefficients were properly calculated.

The first calculation is based on the Poynting flux using orthogonality of the basis set to perform the integral:

$$
P_{E \times B}^{F}=\frac{1}{2 \mu_{0}} \operatorname{Re} \iint\left(\vec{E} \times \vec{B}^{*}\right)_{x}(x=0) d y d z
$$

where $E$ is constructed at $x=0$ using Eq. (14) and the coefficients from the Appendix and applying the example of Eq. (2). Faraday's law was used to construct $B^{*}$ in Fourier space.

The second calculation is based on evaluation of

$$
P_{E \cdot J}=\frac{1}{2} \int_{0}^{d+a_{w}} \int_{-\pi a}^{\pi a} \int_{-\pi R}^{\pi R} \vec{E} \cdot \vec{J}^{*} d z d y d x
$$

where analytic integration in the $x$-direction is performed using Eqs (9) and (14) and the Appendix with numerical summation of the resulting coefficients. Conservation of energy requires that the real part of $P_{E \cdot J}=P_{E \times B}^{F}$. The imaginary part of $P_{E \cdot J}$ is the reactive or recirculating power.

The third calculation involves the reconstructed electric and magnetic fields in real space. Carrying out the surface integral of the Poynting flux numerically gives

$$
\begin{aligned}
P_{E \times B}^{R} & =\frac{1}{2 \mu_{0}} \sum_{i=-I}^{I} \sum_{j=-J}^{J} \\
& \times\left(\vec{E} \times \vec{B}^{*}\right)_{x}^{i j}(x=0) \Delta y \Delta z
\end{aligned}
$$

where the values are from the same grid as that used to plot the real-space reconstruction. In this equation, $\Delta y$ and $\Delta z$ are the real-space step sizes for the numerical grid.

The results from the first and second methods for the (typical) example considered in Section 7 agree to 13 significant digits using IEEE standard double-precision arithmetic (approximately 16 digits of accuracy), indicating the integrity of the coding. The third calculation depends on the range of integration considered in the reconstruction and the grid spacing, as well as the directionality of the launched power. 


\section{RESULTS}

The MAntIS code has keen used to model very complicated antenna structures, such as the folded waveguide $[5,6]$, by superimposing many basic current elements. However, to illustrate the use of some of the code's features, a simple current strap with feeders and finite phase velocity $v_{\phi}$ along the main strap is presented here. This particular example stems from early design considerations for a simple loop antenna to be used in the Frascati Tokamak Upgrade (FT-U) experiment in Frascati, Italy. The design parameters are given in Table I; the coupled power through a single port was required to be between 0.5 and $1 \mathrm{MW}$.

The modelling was performed using 201 poloidal and 401 toroidal Fourier modes, where $y$ is the poloidal direction (along the main strap) and $z$ is the toroidal direction (along the static magnetic field). The cold deuterium plasma density profile used in ORION-1D to calculate the impedance tensor, $\overrightarrow{\vec{Z}}$, can be described by the following analytic formula:

$$
\begin{aligned}
& n=\left(n_{0}-n_{e}\right)\left(1-\frac{r^{2}}{a_{s}^{2}}\right)+n_{e}, \quad r \leq a_{s} \\
& n=n_{e} \exp \left[\left(r-a_{s}\right) / \lambda_{s}\right], \quad a_{s}<r<a_{p}
\end{aligned}
$$

where values for the parameters are given in Table I. Two loop antennas that were fed separately and stacked poloidally were considered so that the RF magnetic field could be maximized. The height of each strap was chosen to roughly match the expected halfwavelength at $\omega=433 \mathrm{MHz}$ for a finite phase velocity of $0.5 \mathrm{c}$ with the maximum current carried at the center of the strap. The finite phase velocity effect is introduced in Eq. (6) by setting $K(y, z)=\cos \left(\omega y / v_{\phi}\right)$. A feeder carrying zero net current at the centre of each strap is required to produce such a current profile but is not explicitly considered in the model. The result of this choice is that the feeders carry only a small fraction of the maximum current density.

For the example considered here, $75 \%$ of the power was accounted for in the range $-0.1 \mathrm{~m}<y, z<0.1 \mathrm{~m}$. The real-space integration is a useful diagnostic of the directionality of the launch power as well as an additional check of the code.

Figures 5 and 6 were generated using Eq. (2) to reconstruct each RF electric and magnetic field component over a range from $-0.1 \mathrm{~m}$ to $0.1 \mathrm{~m}$ in both the $y$ - and $z$-directions with 72 points in each direction. The fields were reconstructed at 11 evenly spaced $x$-locations between the plasma interface and the conducting backwall. The geometry function surface was constructed by normalizing to the peak value in the feeder regions and choosing the nearest $x$-reconstruction plane for the main strap. The modulus of the RF fields and the isosurfaces of the modulus were generated using Data Explorer on a RISC System 6000 workstation with color-to-greyscale conversion and image enhancement using the PBMPLUS package (C) 1989,1991 by Jef Poskanzer).

Figure 5 shows that the maximum RF magnetic field occurs near the centre of the main current strap, where the current is at a maximum. Figure 6 shows the electric field isosurfaces that occur between the current strap and the plasma. The electric field is predominantly in the radial $(x)$ direction, pointing from the strap to the plasma, near the top of the main strap and in the opposite direction near the bottom. These fields are primarily capacitive because of the charge buildup near the ends of the strap caused by the 
finite phase velocity. A real antenna has a Faraday shield, consisting of parallel bars placed roughly perpendicular to the strap, to isolate this electrostatic field from the plasma and reduce nonlinear effects. The electric field in the model does not account for the Faraday shield explicitly; however, the plasma is a very good conductor in the direction of the static magnetic field lines (roughly toroidal) and behaves in a manner very similar to ? Faraday shield for the plasma density that was used.

If we consider the plasma and current strap with a vacuum separation as a cansitior, the electric field that would result from a Faraday shield of finite thickness, $d_{f}$, is increased by a factor of roughly $d /\left(d-d_{f}\right)$. The coupled power corresponding to a maximum electric field of $1.74 \times 10^{4} \mathrm{~V} / \mathrm{m}$ is calculated to be $12.8 \mathrm{~W}$ for $d_{f}=0$. With the power normalized to the estimated maximum tolerable electric field for arcing of $2 \times 10^{6} \mathrm{~V} / \mathrm{m}$ and a Faraday shield with $d_{f}=0.01 \mathrm{~m}$, the maximum coupled power using the structure is predicted by the model to be $42 \mathrm{~kW}$. Thus, the modelling shows that two such antennas that are separately driven and poloidally stacked in the port of the machine will couple less than $100 \mathrm{~kW}$. This coupling is far below the design requirement and demonstrates the difficulties involved in using a standard loop antenna design to couple power to the FT-U plasma. Extensive modelling using MAntIS for a folded waveguide launching structure has shown the folded waveguide technology to be a possible solution to the problem of large electric fields when coupling RF power to the FT-U plasma $[5,6]$.

\section{APPENDIX}

Two combinations of feeder type and backwall boundary have been tested in MAntIS for two different types of modelling. Both combinations satisfy Eq. (7b).

The first combination has constant current along each feeder, $j_{0}=1$ and $j_{p \neq 0}=0$, with boundary conditions of $E_{y \mathrm{I}}^{m, n}\left(x=d+a_{w}\right)=0$ and $E_{z \mathrm{I}}^{m, n}\left(x=d+a_{w}\right)=0$ (conducting backwall). This combination has been used for typical loop antenna modelling, as demonstrated in Section 7.

The second combination has both standing and travelling waves for the current along the feeder such that $j_{1}=(\zeta+1 / 2), j_{-1}=-(\zeta-1 / 2)$, and $j_{p \neq \pm 1}=0$. The boundary conditions used with this feeder type are

$$
\begin{aligned}
E_{y \mathrm{I}}^{m, n}\left(x=d+a_{w}\right)= & \frac{i \mu_{0} \pi c^{2}}{\omega a_{w}} \frac{m}{a} \mathcal{J}_{x}^{m, n} \\
& \times \frac{-2 \zeta}{\left(k_{\perp}^{2 m, n}-\pi^{2} / a_{w}^{2}\right)} \\
E_{z \mathrm{I}}^{m, n}\left(x=d+a_{w}\right)= & \frac{i \mu_{0} \pi c^{2}}{\omega a_{w}} \frac{n}{R} \mathcal{J}_{x}^{m, n} \\
& \times \frac{-2 \zeta}{\left(k_{\perp}^{2 m, n}-\pi^{2} / a_{w}^{2}\right)} .
\end{aligned}
$$

where $\mathcal{J}_{\mathfrak{x}}^{m, n}$ is defined by Eq. (9b), and $\zeta$ is a travelling wave parameter. This boundary condition is equivalent to a conducting backwall for standing waves where $\zeta=0$, but permits power carried by the travelling wave to flow through the backwall when $\zeta \neq 0$. This 
combination has been used to model folded waveguide antenna structures $[5,6]$ primarily for $\zeta=0$.

The fields in Region II are

$$
\begin{aligned}
& E_{y \mathrm{II}}^{m, n}=A_{\mathrm{I}}^{m, n}\left\{\exp \left[i k_{\perp}^{m, n}\left(x-a_{w}-d\right)\right]-\exp \left[-i k_{\perp}^{m, n}\left(x-a_{w}-d\right)\right]\right\} \\
& +\frac{\omega \mu_{0}}{2 k_{\perp}^{m, n}}\left(\mathcal{K}_{y}^{m, n}+\frac{\pi}{a_{w} \omega \mu_{0}} \mathcal{P}_{y}^{m, n}\right) \\
& \times\left\{\exp \left[i k_{\perp}^{m, n}(x-d)\right]-\exp \left[-i k_{\perp}^{m, n}(x-d)\right]\right\} \\
& +\mathcal{P}_{y}^{m, n} \zeta\left\{\exp \left[i k_{\perp}^{m, n}(x-d)\right]+\exp \left[-i k_{\perp}^{m, n}(x-d)\right]\right\} \\
& E_{z \mathrm{II}}^{m, n}=C_{\mathrm{I}}^{m, n}\left\{\exp \left[i k_{\perp}^{m, n}\left(x-a_{w}-d\right)\right]-\exp \left[-i k_{\perp}^{m, n}\left(x-a_{w}-d\right)\right]\right\} \\
& +\frac{\omega \mu_{0}}{2 k_{\perp}^{m, n}}\left(\mathcal{K}_{z}^{m, n}+\frac{\pi}{a_{w} \omega \mu_{0}} \mathcal{P}_{z}^{m, n}\right) \\
& \times\left\{\exp \left[i k_{\perp}^{m, n}(x-d)\right]-\exp \left[-i k_{\perp}^{m, n}(x-d)\right]\right\} \\
& +\mathcal{P}_{z}^{m, n} \zeta\left\{\exp \left[i k_{\perp}^{m, n}(x-d)\right]+\exp \left[-i k_{\perp}^{m, n}(x-d)\right]\right\}
\end{aligned}
$$

where

$$
\begin{aligned}
\mathcal{P}_{y}^{m, n} \equiv & \frac{\mu_{0} \pi c^{2} / \omega a_{w}}{k_{\perp}^{2 m, n}-\pi^{2} / a_{w}^{2}} \frac{m}{a} \sum_{m^{\prime}, n^{\prime}} K^{m^{\prime}, n^{\prime}} f^{m-m^{\prime}, n-n^{\prime}} \\
& \times\left(\frac{m-m^{\prime}}{a} \sin \theta+\frac{n-n^{\prime}}{R} \cos \theta\right)
\end{aligned}
$$

and

$$
\mathcal{P}_{z}^{m, n} \equiv \frac{n a}{m R} \mathcal{P}_{y}^{m, n}
$$

The solutions for the Region I coefficients, $A_{\mathrm{I}}^{m, n}$ and $C_{\mathrm{I}}^{m, n}$, are compactly written as

$$
\begin{aligned}
A_{\mathrm{I}}^{m, n}= & \Gamma_{a y}^{m, n} K_{y, f f}^{m, n}+\Gamma_{a z}^{m, n} K_{z_{\text {eff }}}^{m, n} \\
& -\zeta\left(\Gamma_{a p y}^{m, n} \mathcal{P}_{y}^{m, n}+\Gamma_{a p z}^{m, n} \mathcal{P}_{z}^{m, n}\right) \\
C_{\mathrm{I}}^{m, n}= & \Gamma_{c y}^{m, n} K_{y, f f}^{m, n}+\Gamma_{c z}^{m, n} K_{z_{\text {eff }}, m}^{m, n} \\
& -\zeta\left(\Gamma_{c p y}^{m, n} \mathcal{P}_{y}^{m, n}+\Gamma_{c p z}^{m, n} \mathcal{P}_{z}^{m, n}\right)
\end{aligned}
$$

where

$$
\begin{aligned}
& \left.i \omega \mu_{0} K_{y_{\text {eff }}^{m, n}}^{m,} \equiv \frac{d \mathrm{P}_{y}^{m, n}}{d x}\right|_{x=d}+i \omega \mu_{0} K_{y}^{m, n} \\
& \left.i \omega \mu_{0} K_{z_{\text {eff }}^{m, n}}^{m, n} \equiv \frac{d \mathrm{P}_{z}^{m, n}}{d x}\right|_{x=d}+i \omega \mu_{0} K_{z}^{m, n}
\end{aligned}
$$

and 


$$
\begin{aligned}
\Gamma_{a y}^{m, n} \equiv & \frac{\omega \mu_{0}}{2 k_{\perp}^{m, n}}\left[\left(\psi_{-}^{m, n}-\alpha_{1}^{m, n} \psi_{+}^{m, n}\right)\right. \\
& \times\left(-\phi_{-}^{m, n}+\beta_{2}^{m, n} \phi_{+}^{m, n}\right) \\
& \left.+\alpha_{2}^{m, n} \beta_{1}^{m, n} \psi_{+}^{m, n} \phi_{+}^{m, n}\right] / \Gamma_{d}^{m, n} \\
\Gamma_{a z}^{m, n} \equiv & \frac{\omega \mu_{0}}{2 k_{\perp}^{m, n}}\left[\beta _ { 1 } ^ { m , n } \left(\psi_{+}^{m, n} \phi_{-}^{m, n}\right.\right. \\
& \left.\left.-\psi_{-}^{m, n} \phi_{+}^{m, n}\right)\right] / \Gamma_{d}^{m, n} \\
\Gamma_{a p y}^{m, n} \equiv & {\left[\left(\alpha_{1}^{m, n} \psi_{-}^{m, n}-\psi_{+}^{m, n}\right)\left(\phi_{-}^{m, n}-\beta_{2}^{m, n} \phi_{+}^{m, n}\right)\right.} \\
\Gamma_{a p z}^{m, n} \equiv & \left.\left.\quad+\alpha_{2}^{m, n} \psi_{-}^{m, n} \beta_{1}^{m, n} \phi_{+}^{m, n}\right] / \Gamma_{d}^{m, n}\left(\psi_{-}^{m, n} \phi_{-}^{m, n}-\psi_{+}^{m, n} \phi_{+}^{m, n}\right)\right] / \Gamma_{d}^{m, n} \\
\Gamma_{c y}^{m, n} \equiv & \frac{\omega \mu_{0}}{2 k_{\perp}^{m, n}}\left[\alpha_{2}^{m n}\left(\psi_{+}^{m, n} \phi_{-}^{m, n}-\phi_{+}^{m, n} \psi_{-}^{m, n}\right)\right] / \Gamma_{d}^{m, n} \\
\Gamma_{c z}^{m, n} \equiv & \frac{\omega \mu_{0}}{2 k_{\perp}^{m, n}}\left[\left(\beta_{2}^{m, n} \psi_{+}^{m, n}-\psi_{-}^{m, n}\right)\right. \\
& \quad \times\left(\phi_{-}^{m, n}-\alpha_{1}^{m, n} \phi_{+}^{m, n}\right) \\
\quad & \left.\quad \beta_{1}^{m, n} \psi_{+}^{m, n} \alpha_{2}^{m, n} \phi_{+}^{m, n}\right] / \Gamma_{d}^{m, n} \\
\Gamma_{c p y}^{m, n} \equiv & {\left[\alpha_{2}^{m, n}\left(\phi_{-}^{m, n} \psi_{-}^{m, n}-\phi_{+}^{m, n} \psi_{+}^{m, n}\right)\right] / \Gamma_{d}^{m, n} } \\
\Gamma_{c p z}^{m, n} \equiv & {\left[\left(\beta_{2}^{m, n} \psi_{-}^{m, n}-\psi_{+}^{m, n}\right)\left(\phi_{-}^{m, n}-\alpha_{1}^{m, n} \phi_{+}^{m, n}\right)\right.} \\
& \left.\quad+\beta_{1}^{m, n} \psi_{-}^{m, n} \alpha_{2}^{m, n} \phi_{+}^{m, n}\right] / \Gamma_{d}^{m, n}
\end{aligned}
$$

with

$$
\begin{aligned}
\Gamma_{d}^{m, n} \equiv & \left(-\phi_{-}^{m, n}+\alpha_{1}^{m, n} \phi_{+}^{m, n}\right)\left(-\phi_{-}^{m, n}+\beta_{2}^{m, n} \phi_{+}^{m, n}\right) \\
\alpha_{1}^{m, n} \equiv & -\frac{1}{\omega \mu_{0} k_{\perp}^{m, n}}\left[\mathcal{Z}_{11}^{m, n}\left(\frac{m n}{a R}\right)\right. \\
& \left.-\left(\frac{w^{2}}{c^{2}}-Z_{12}^{m, n} \frac{n^{2}}{R^{2}}\right)\right] \\
\beta_{1}^{m, n} \equiv & \frac{1}{\omega \mu_{0} k_{\perp}^{m, n}}\left[\mathcal{Z}_{11}^{m, n}\left(\frac{w^{2}}{c^{2}}-\frac{m^{2}}{a^{2}}\right)\right. \\
\alpha_{2}^{m, n} \equiv & \frac{1}{\omega \mu_{0} k_{\perp}^{m, n}}\left[Z_{21}^{m, n}\left(\frac{m n}{a R}\right)\right. \\
& \left.-Z_{22}^{m, n}\left(\frac{m n}{a R}\right)\right] \\
\beta_{2}^{m, n} \equiv & \frac{1}{\omega \mu_{0} k_{\perp}^{m, n}}\left[Z_{21}^{m, n}\left(\frac{w^{2}}{c^{2}}-\frac{n^{2}}{R^{2}}\right)\right] \\
& \left.-Z_{22}^{m, n}\left(\frac{m n}{a R}\right)\right]
\end{aligned}
$$




$$
\begin{aligned}
\phi_{ \pm}^{m, n} & \equiv \exp \left[i k_{\perp}^{m, n}\left(a_{w}+d\right)\right] \pm \exp \left[-i k_{\perp}^{m, n}\left(a_{w}+d\right)\right] \\
\psi_{ \pm}^{m, n} & \equiv \exp \left(i k_{\perp}^{m, n} d\right) \pm \exp \left(-i k_{\perp}^{m, n} d\right)
\end{aligned}
$$

In terms of these Region I coefficients, the electric fields in Region I along with the particular solutions for existing feeder options are given by Eq. (10), and the fields in Region II are given by Eq. (14). 


\section{REFERENCES}

11] GOULDING, R.H., BAITY, F.W., BATCHELOR, D.B., et al., in Riadio Frequency Power in Plasmas (Proc. 9th Topical Conf. Charleston, 1991) (BATCHELOR, D.B., ed.), American Institute of Physics, New York (1992) 287 (AIP Conf. Proc. 244 (1992) 287].

[2] JAEGER, E.F., BATCHELOR, D.B., WEITZNER, H., Nucl. Fusion 28 (1988) 53.

[3] BRAMBILla, M., Nucl. Fusion 28 (1988) 549.

[4] THEILHABER, K., JACQUINOT, J., Nucl. Fusion 24 (1984) 541.

[5] HASTE, G.R., BAITY, F.W., BARBER, G.L., et al., The folded waveguide: a high frequency rf launcher, submitted to Fusion Eng. Des.

[6] CARTER, M.D., BAITY, F.W., BATCHELOR, D.B., et al., in Radio Frequency Power in Plasmas (Proc. 9th Topical Conf. Charleston, 1991) (BATCHELOR, D.B., ed.), American Institute of Physics, New York (1992) 164 [AIP Conf. Proc. 244 (1992) 164]. 
TABLE I. DESIGN PARAMETERS FOR FT-U ANTENNA

\begin{tabular}{ll}
\hline Port size & $0.07 \times 0.4 \mathrm{~m}$ \\
Major radius, $R$ & $0.93 \mathrm{~m}$ \\
Minor radius, $a$ & $0.35 \mathrm{~m}$ \\
Plasma minor radius, $a_{p}$ & $0.3 \mathrm{~m}$ \\
Plasma minor radius for & \\
$\quad$ slab calculation, $a_{s}$ & $0.288 \mathrm{~m}$ \\
Distance from current strap & \\
$\quad$ to back wall, $a_{w}$ & $0.13 \mathrm{~m}$ \\
Distance from plasma-vacuum & \\
interface to current strap, $d$ & $0.02 \mathrm{~m}$ \\
Neutral density, $n_{0}$ & $1 \times 10^{20} \mathrm{~m}^{-3}$ \\
Electron density, $n_{e}$ & $1.5 \times 10^{19} \mathrm{~m}^{-3}$ \\
Resonant frequency, $\omega$ & $433 \mathrm{MHz}^{-3}$ \\
Effective wavelength, $\lambda_{s}$ & $0.06 \mathrm{~m}$ \\
Finite phase velocity, $v_{\phi}$ & $0.5 \mathrm{c}$ \\
Antenna model parameters & \\
$\quad$ Angle of rotation, $\Theta$ & $90^{\circ}$ \\
$\quad$ Current element width, $\alpha$ & 0.03 \\
$\quad$ Current element length, $\beta$ & 0.18 \\
\hline
\end{tabular}




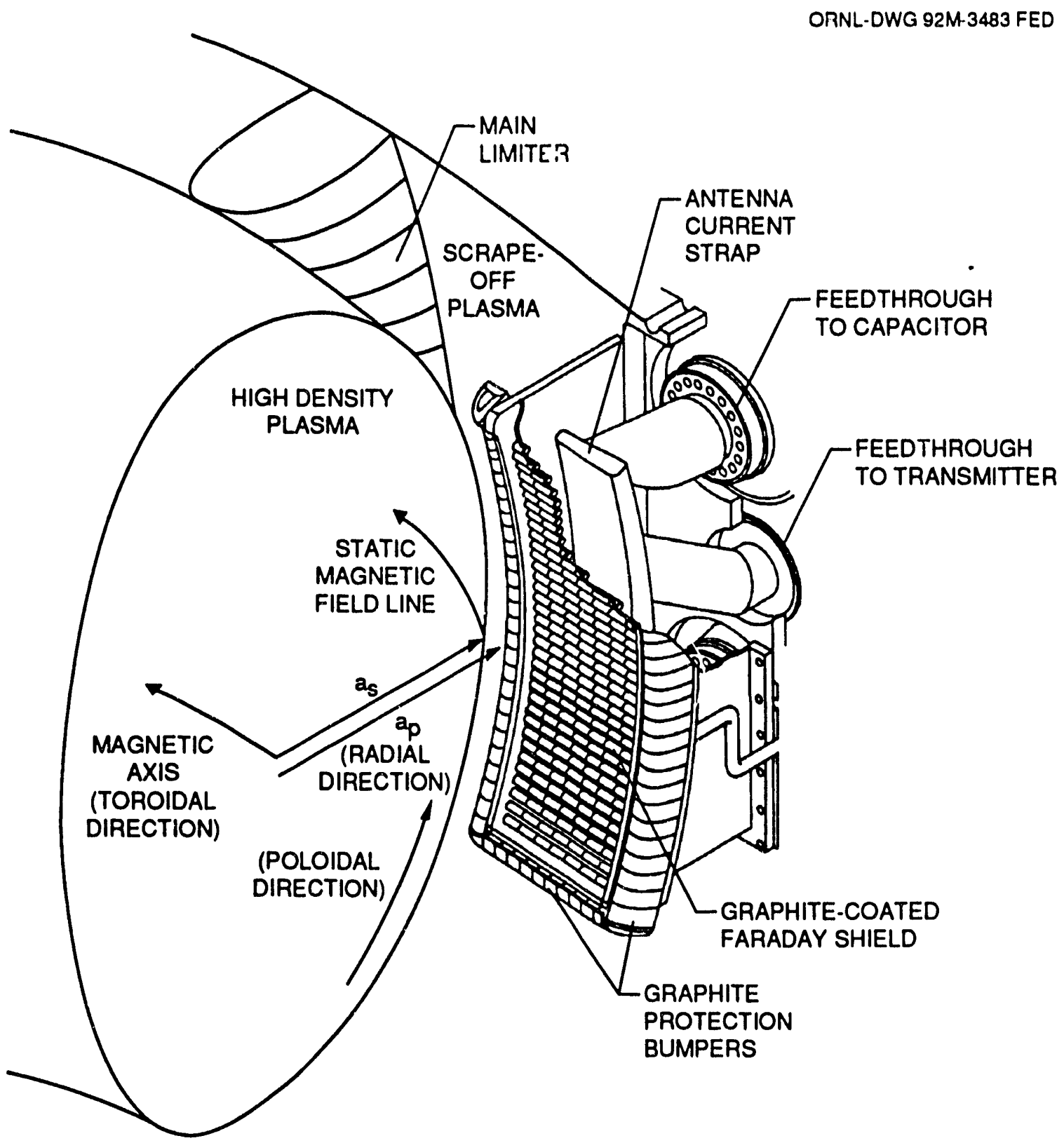

Figure 1: Schematic of a typical loop antenna design for use in a tokamak fusion experiment. 


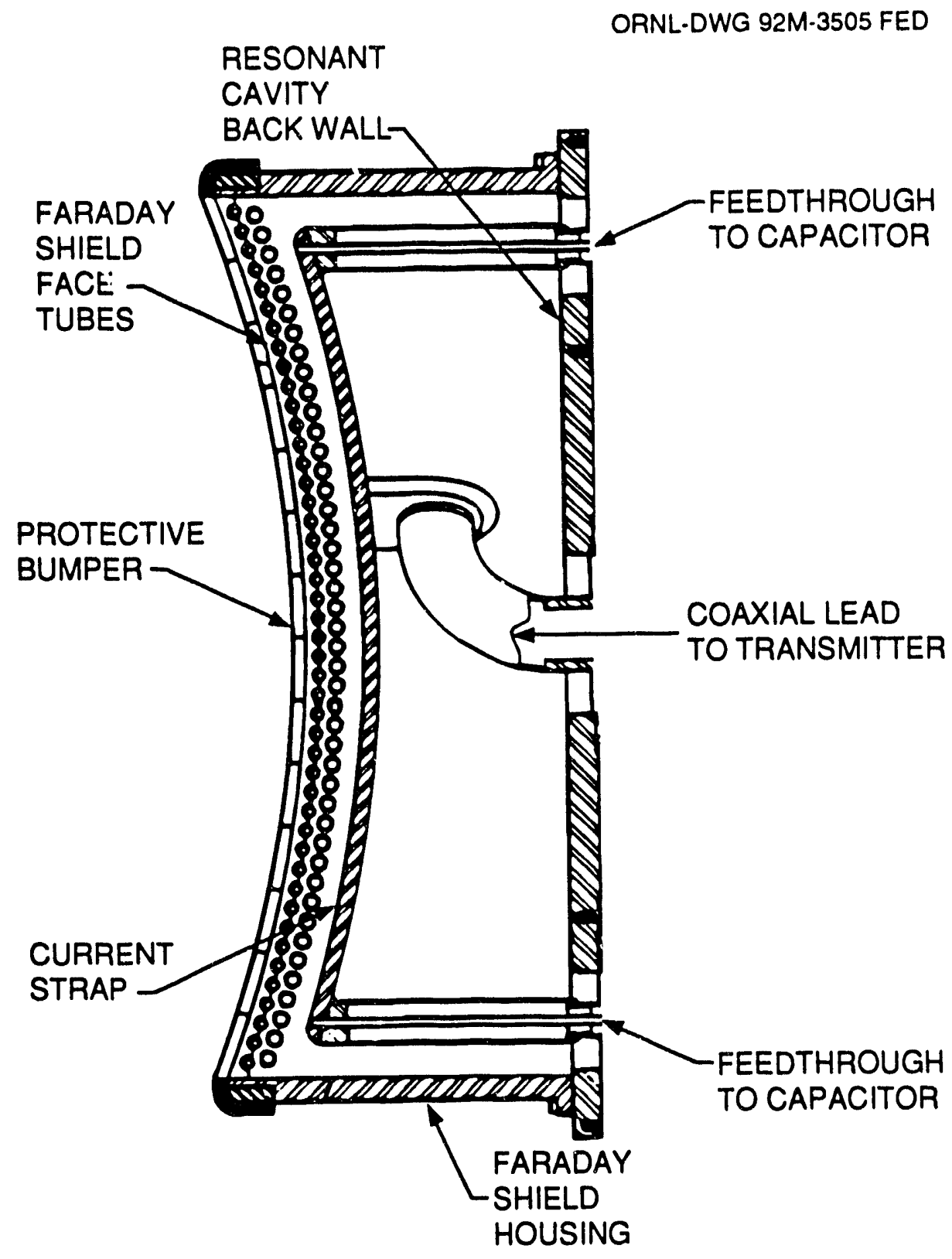

Figure 2: Parts of a loop antenna that MAntIS models. Note that the modelling of the Faraday screen is not explicit. 

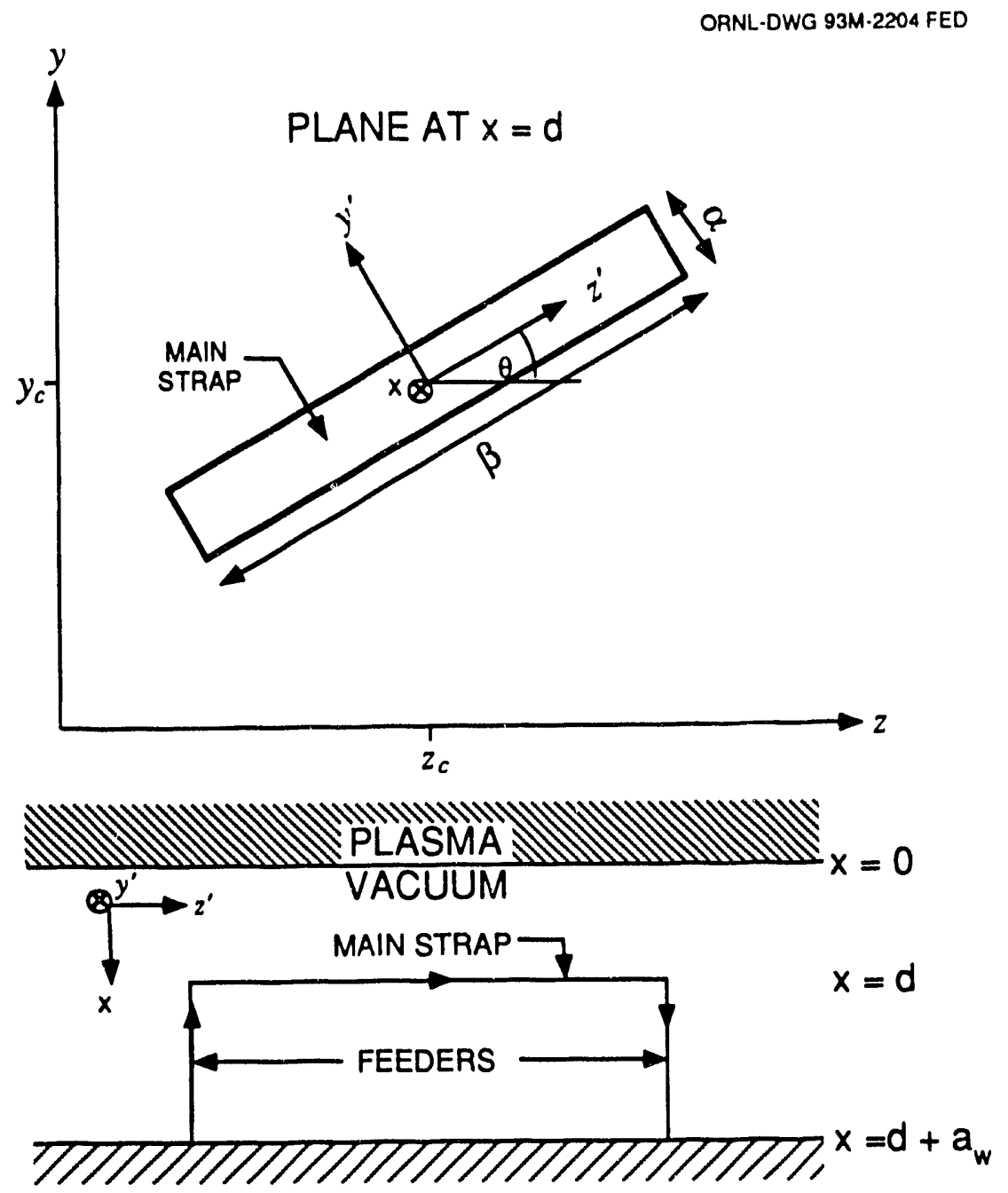

CONDUCTING BACKWALL

Figure 3: Geometry of basic elements used by MAntIS in two views, showing the orientation of the driven currents, the matching regions and the coordinate system. 
ORNL-DWG 93-2205 FED

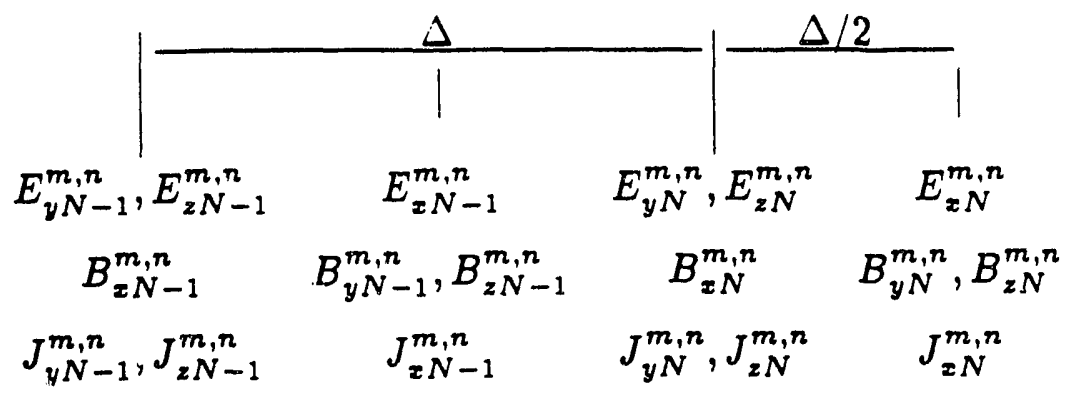

Figure 4: Grid used for finite differencing in the ORION-1D code to calculate the impedance match between the plasma and vacuum regions. 


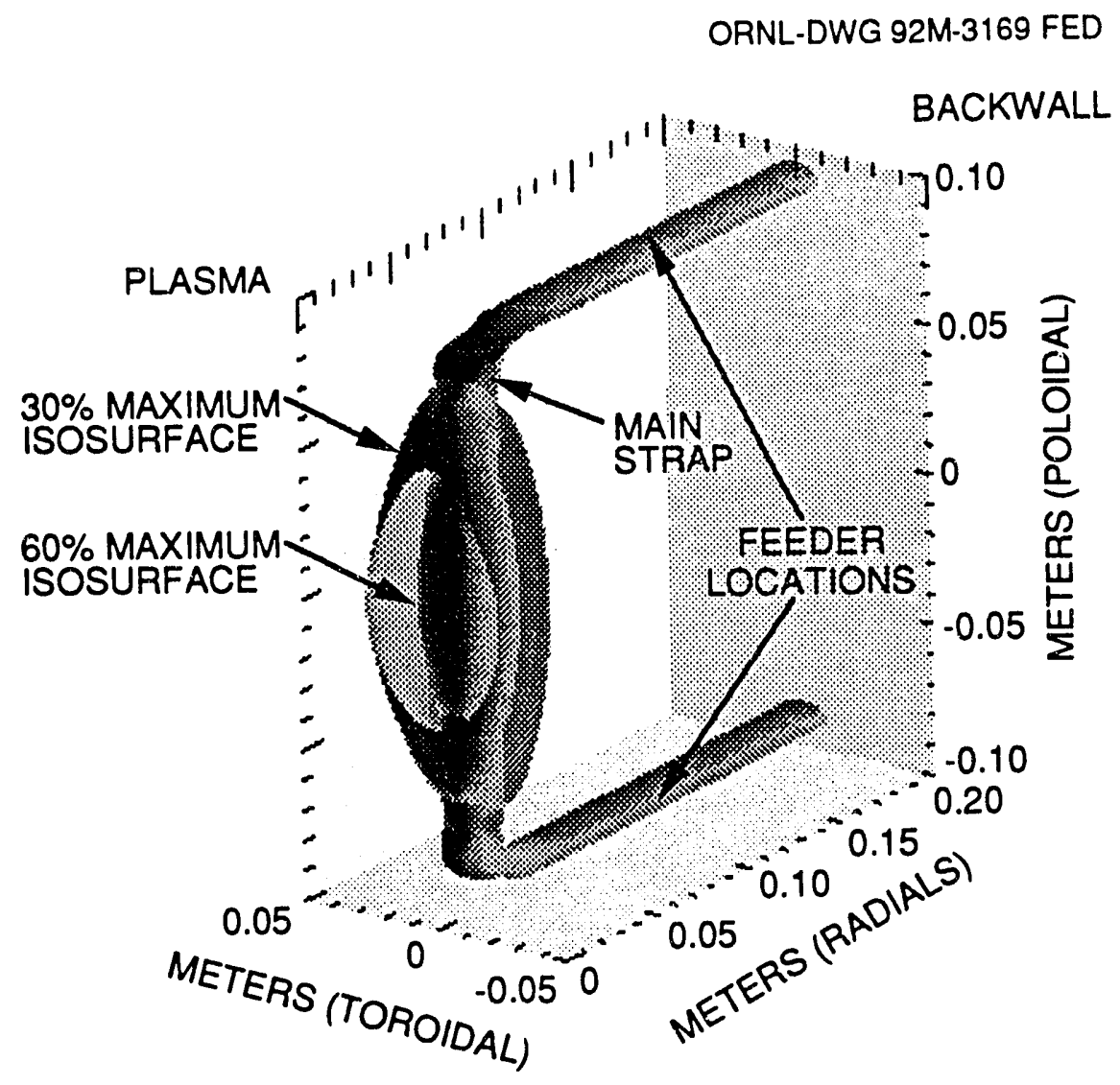

Figure 5: Inverse Fourier reconstruction of solutions in real space showing isosurfaces of constant RF magnetic field amplitude along with the surface of the geometry function $v_{\phi}=0.5 c$ in FT-U. 


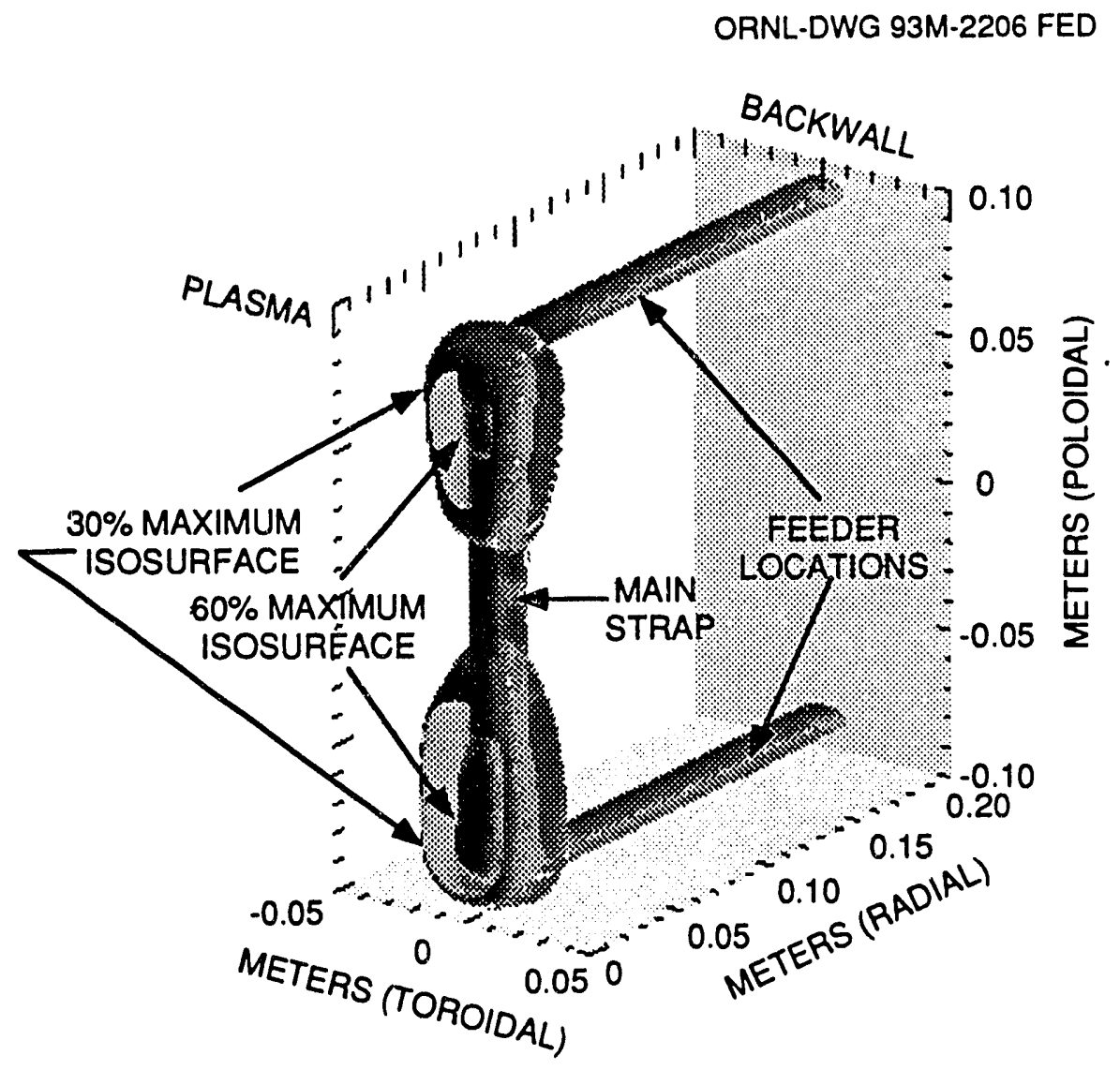

Figure 6: As in Fig. 5 except that isosurfaces of constant electric field amplitude are shown. 


\section{INTERNAL DISTRIBUTION}

1. Director, ORNL Fusion Energy Division

2. C. C. Baker

3. M. J. Saltmarsh

4. L. A. Berry

5. B. A. Carreras

6. R. A. Dory

7. J. L. Dunlap

8. H. H. Haselton

9. M. S. Lubell

10. T. E. Shannon

11. R. P. Leinius

12-13. Laboratory Records Department

14. Laboratory Records, ORNL-RC

15-16. Central Research Library

17. Document Reference Section

18. Fusion Energy Division Library

19-20. ET/FE Division Publications Office

21. ORNL Patent Office
22-26. D. B. Batchelor

27-31. M. D. Carter

32. F. W. Baity

33. T. S. Bigelow

34. R. C. Goldfinger

35. R. H. Goulding

36. S. P. Hirshman

37. D. J. Hoffman

38. W. A. Houlberg

39. E. F. Jaeger

40. S. L. Milora

41. D. A. Rasmussen

42. P. M. Ryan

43. D. C. Stallings

44. D. W. Swain

45. J. H. Whealton

46. J. J. Yugo

\section{EXTERNAL DISTRIBUTION}

47. Office of the Assistant Manager for Energy Research and Development, U.S. Department of Energy Field Office, Oak Ridge, P.O. Box 2000, Oak Ridge, TN 37831

48. N. A. Davies, Director, Office of Fusion Energy, Office of Energy Research, ER-50 Germantown, U.S. Department of Energy, Washington, DC 20545

49. M. Roberts, International Programs, Office of Fusion Energy, Office of Energy Research, ER-52 Germantown, U.S. Department of Energy, Washington, DC 20545

50. D. E. Baldwin, Lawrence Livermore National Laboratory, P.O. Box 5511, Livermore, CA 94550

51. R. W. Conn, Mechanical, Aerospace, and Nuclear Engineering Department, 6291 Boelter Hall, University of California, Los Angeles, CA 90024-1597

52. P. C. Liewer, MS 138-208, Jet Propulsion Laboratory, 4800 Oak Grove Drive, Pasadena, CA 91109

53. D. Sigmar, Plasma Fusion Center, Massachusetts Institute of Technology, 167 Albany St., NW16-288, Cambridge, MA 02139

54. K. I. Thomassen, L-637, Lawrence Livermore National Laboratory, P.O. Box 5511, Livermore, CA 94550

55. J. D. Callen, Department of Nuclear Engineering, University of Wisconsin, Madison, WI 53706-1687

56. S. O. Dean, Fusion Power Associates, Inc., 2 Professional Drive, Suite 248, Gaithersburg, MD 20879

57. H. K. Forsen, Bechtel Group, Inc., Research Engineering, P.O. Box 3965, San Francisco, CA 94119 
58. R. W. Gould, Department of Applied Physics, California Institute of Technology, Pasadena, CA 91125

59. R. A. Gross, Plasma Research Laboratory, Columbia University, New York, NY 10027

60. R. J. Hawryluk, Plasma Physics Laboratory, Princeton University, P.O. Box 451, Princeton, NJ 08543

61. D. M. Meade, Plasma Physics Laboratory, Princeton University, P.O. Box 451, Princeton, NJ 08543

62. W. M. Stacey, School of Nuclear Engineering and Health Physics, Georgia Institute of Technology, Atlanta, GA 30332

63. D. Steiner, Nuclear Engineering Department, NES Building, Tibbetts Avenue, Rensselaer Polytechnic Institute, Troy, NY 12181

64. R. Varma, Physical Research Laboratory, Navrangpura, Ahmedabad 380009, India

65. Bibliothek, Max-Planck Institut für Plasmaphysik, Boltzmannstrasse 2, D-8046 Garching, Federal Republic of Germany

66. Bibliothek, Institut für Plasmaphysik, KFA Jülich GmbH, Postfach 1913, D-5170 Jülich, Federal Republic of Germany

67. Bibliothek, KfK Karlsruhe GmbH, Postfach 3640, D-7500 Karlsruhe 1, Federal Republic of Germany

68. Bibliotheque, Centre de Recherches en Physique des Plasmas, Ecole Polytechnique Fédérale de Lausanne, 21 Avenue des Bains, $\mathrm{CH}-1007$ Lausanne, Switzerland

69. R. Aymar, CEN/Cadarache, Departement de Recherches sur la Fusion Contrôlée, F-13108 Saint-Paul-lez-Durance Cedex, France

70. Bibliothèque, CEN/Cadarache, F-13108 Saint-Paul-lez-Durance Cedex, France

71. Library, JET Joint Undertaking, Abingdon, Oxfordshire OX14 3EA, England

72. Library, FOM-Instituut voor Plasmafysica, Rijnhuizen, Edisonbaan 14, 3439 MN Nieuwegein, The Netherlands

73. Library, National Institute for Fusion Science, Chikusa-ku, Nagoya 464-01, Japan

74. Library, International Centre for Theoretical Physics, P.O. Box 586, I-34100 Trieste, Italy

75. Library, Centro Richerche Energia Frascati, C.P. 65, I-00044 Frascati (Roma), Italy

76. Library, Plasma Physics Laboratory, Kyoto University, Gokasho, Uji, Kyoto 611, Japan

77. Plasma Research Laboratory, Australian National University, P.O. Box 4, Canberra, A.C.T. 2601, Australia

78. Library, Japan Atomic Energy Research Institute, Naka Fusion Research Establishment, 801-1 Mukoyama, Naka-machi, Naka-gun, Ibaraki-ken, Japan

79. G. A. Eliseev, I. V. Kurchatov Institute of Atomic Energy, P.O. Box 3402, 123182 Moscow, Russia

80. V. A. Glukhikh, Scientific-Research Institute of Electro-Physical Apparatus, 188631 St. Petersburg, Russia

81. I. Shpigel, Institute of Generai Physics, U.S.S.R. Academy of Sciences, Ulitsa Vavilova 38, Moscow, Russia

82. D. D. Ryutov, Institute of Nuclear Physics, Siberian Academy of Sciences, Sovetskaya St. 5, 630090 Novosibirsk, Russia

83. O. Pavlichenko, Kharkov Physical-Technical Institute, Academical St. 1, 310108 Kharkov, Ukraine

84. Deputy Director, Southwestern Institute of Physics, P.O. Box 15, Leshan, Sichuan, China (PRC)

85. Director, The Institute of Plasma Physics, P.O. Box 1126, Hefei, Anhui, China (PRC)

86. R. H. McKnight, Experimental Plasma Research Branch, Division of Development and Technology, Office of Fusion Energy, Office of Energy Research, ER-542 Germantown, U.S. Department of Energy, Washington, DC 20545

87. D. Crandall, Director, Division of Applied Plasma Physics, Office of Fusion Energy, Office of Energy Research, ER-54 Germantown, U.S. Department of Energy, Washington, DC 20545 
88. E. Oktay, Division of Confinement Systems, Office of Fusion Energy, Office of Energy Research, ER-55 Germantown, U.S. Department of Energy, Washington, DC 20545

89. W. Sadowski, Fusion Theory and Computer Services Branch, Division of Applied Plasma Physics, Office of Fusion Energy, Office of Energy Research, ER-541 Germantown, U.S. Department of Energy, Washington, DC 20545

90. R. E. Mickens, Atlanta University, Department of Physics, Atlanta, GA 30314

91. M. N. Rosenbluth, University of California at San Diego, La Jolla, CA 92037

92. D. Schnack, SAIC, 10260 Campus Point Drive, San Diego, CA 92121

93. Duk-In Choi, Department of Physics, Korea Advanced Institute of Science and Technology, P.O. Box 150, Chong Ryang-Ri, Seoul, Korea

94. Library of Physics Department, University of Ioannina, Ioannina, Greece

95. C. De Palo, Library, Associazione EURATOM-ENEA sulla Fusione, CP 65, I-00044 Frascati (Roma), Italy

96. Laboratorio Associado de Plasma, Instituto Nacional de Pesquisas Espaciais, Caixa Postal 515, 122201, Sao Jose dos Campos, SP, Brazil

97. Theory Department Read File, c/o D. W. Ross, University of Texas, Institute for Fusion Studies, Austin, TX 78712

98. Theory Department Read File, c/o R. Parker, Director, Plasma Fusion Center, NW 16-202, Massachusetts Institute of Technology, Cambridge, MA 02139

99. Theory Department Read File, c/o R. White, Plasma Physics Laboratory, Princeton University, P.O. Box 451, Princeton, NJ 08543

100. Theory Department Read File, c/o L. Kovrizhnykh, Lebedev Institute of Physics, Academy of Sciences, 53 Leninsky Prospect, 117924 Moscow, Russia

101. Theory Department Read File, c/o B. B. Kadomtsev, I. V. Kurchatov Institute of Atomic Energy, P.O. Box 3402, 123182 Moscow, Russia

102. Theory Department Read File, c/o T. Kamimura, National Institute for Fusion Studies, Nagoya 464, Japan

103. Theory Department Read File, c/o E. Maschke, Departemente de Recherches sur la Fusion Controlée, CEN/Cadarache, F-13108 Saint-Paul-lez Durance, France

104. Theory Department Read File, c/o D. Düchs, JET Joint Undertaking, Abingdon, Oxfordshire OX14 3EA, United Kingdom

105. Theory Department Read File, c/o R. Briscoe, Culham Laboratory, Abingdon, Oxfordshire OX14 3DB, United Kingdom

106. Theory Department Read File, c/o D. Biskamp, Max-Planck-Institut fur Plasmaphysik, Boltzmannstrasse 2, D-8046 Garching, Federal Republic of Germany

107. Theory Department Read File, c/o T. Takeda, Japan Atomic Energy Research Institute, Tokai Fusion Research Establishment, Tokai-mura, Naka-gun, Ibaraki-ken, Japan

108. Theory Department Read File, c/o J. Greene, General Atomics, P.O. Box 85608, San Diego, CA 92186

109. Theory Department Read File, c/o R. Cohen, Lawrence Livermore National Laboratory, P.O. Box 5511, Livermore, CA 94550

110. Theory Department Read File, c/o R. Gerwin, CTR Division, Los Alamos National Laboratory, P.O. Box 1663, Los Alamos, NM 87545

111. R. Aamodt, Lodestar Research Corporation, 2400 Central Avenue, Boulder, CO 80301

112. K. Appert, Centre de Recherches en Physique des Plasmas, Ecole Polytechnique Fédérale de Lausanne, 21 Avenue des Bains, $\mathrm{CH}-1007$ Lausanne, Switzerland

113. M. Ballico, Max-Planck Institut für Plasmaphysik, Boltzmannstrasse 2,

D-8046 Garching, Federal Republic of Germany

114. E. Barbato, Associazione EURATOM-ENEA sulla Fusione, Centro Richerche Energia Frascati, C.P. 65, I-00044 Frascati (Roma), Italy

115 A. Bécoulet, Association EURATOM-CEA sur la Fusion Controlée, Centre d'Etudes Nucléaires de Cadarache, B. P. No. 1, F-13108 Saint Paul lez Durances, France 
116. S. Bernabei, Plasma Physics Laboratory, Princeton University, P.O. Box 451, Princeton, NJ 08543

117. A. Bers, Plasma Fusion Center, Massachusetts Institute of Technology, 38-260, Cambridge, MA 02139

118. V. Bhatnagar, JET Joint Undertaking, Abingdon, Oxfordshire OX14 3EA, England

119. P. T. Bonoli, Plasma Fusion Center, Massachusetts Institute of Technology, 175 Albany St., NW17-115, Cambridge, MA 02139

120. M. Brambilla, Max-Planck Institut für Plasmaphysik, Boltzmannstrasse 2, D-8046 Garching, Federal Republic of Germany

121. Dr. Vincent Chan, General Atomics, P. O. Box 85608, San Diego, CA 92186

122. D. Ehst, Building 205, Argonne National Laboratory, 9700 S. Cass Avenue, Argonne, IL 60538

123. D. Faulconer, Laboratoire de Physique des Plasmas, EURATOM-Etat Belge, Ecole Royale Militaire, Avenue de la Renaissance 30, 1040 Brussels, Belgium

124. D. J. Gambier, JET Joint Undertaking, Abingdon, Oxfordshire OX14 3EA, England

125. S. N. Golovato, Plasma Fusion Center, Massachusetts Institute of Technology, 175 Albany Street, NW17-113, Cambridge, MA 02139

126. W. Grossman, SAIC, 1710 Goodridge Drive, McLean, VA 22102

127. W. Ho, SAIC, 10260 Campus Point Drive, San Diego, CA 92121

128. J. Hosea, Plasma Physics Laboratory, Princeton University, P.O. Box 451, Princeton, NJ 08543

129. Kwok Ko, Stanford Linear Accelerator Center, P.O. Box 4349, Stanford, CA 94309

130. C. C. Petty, General Atomics, P.O. Box 85608, San Diego, CA 92186

131. C. K. Phillips, Plasma Physics Laboratory, Princeton University, P.O. Box 451, Princeton, NJ 08543

132. M. Porkolab, Plasma Fusion Center, Massachusetts Institute of Technology, 175 Albany Street, NW 17-119, Cambridge, MA 02139

133. R. Prater, General Atomics, P.O. Box 85608, San Diego, CA 92186

134. J. Scharer, Electrical and Computer Engineering Department, 1500 Johnson Drive, University of Wisconsin, Madison, WI 53706

135. D. C. Stevens, Courant Institute for Mathematical Sciences, New York University, 251 Mercer Street, New York, NY 10001

136. P. E. Vandenplas, Laboratoire de Physique des Plasmas, EURATOM-Etat Belge, Ecole Royale Militaire, Avenue de la Renaissance 30, 1040 Brussels, Belgium

137. H. Weitzner, Courant Institute for Mathematical Sciences, New York University, 251 Mercer Street, New York, NY 10001

138. J. R. Wilson, Plasma Physics Laboratory, Princeton University, P.O. Box 451, Princeton, NJ 08543

139-86. Given distribution according to OSTI-4500, Magnetic Fusion Energy (Category Distribution UC-427, Theoretical Plasma Physics) 

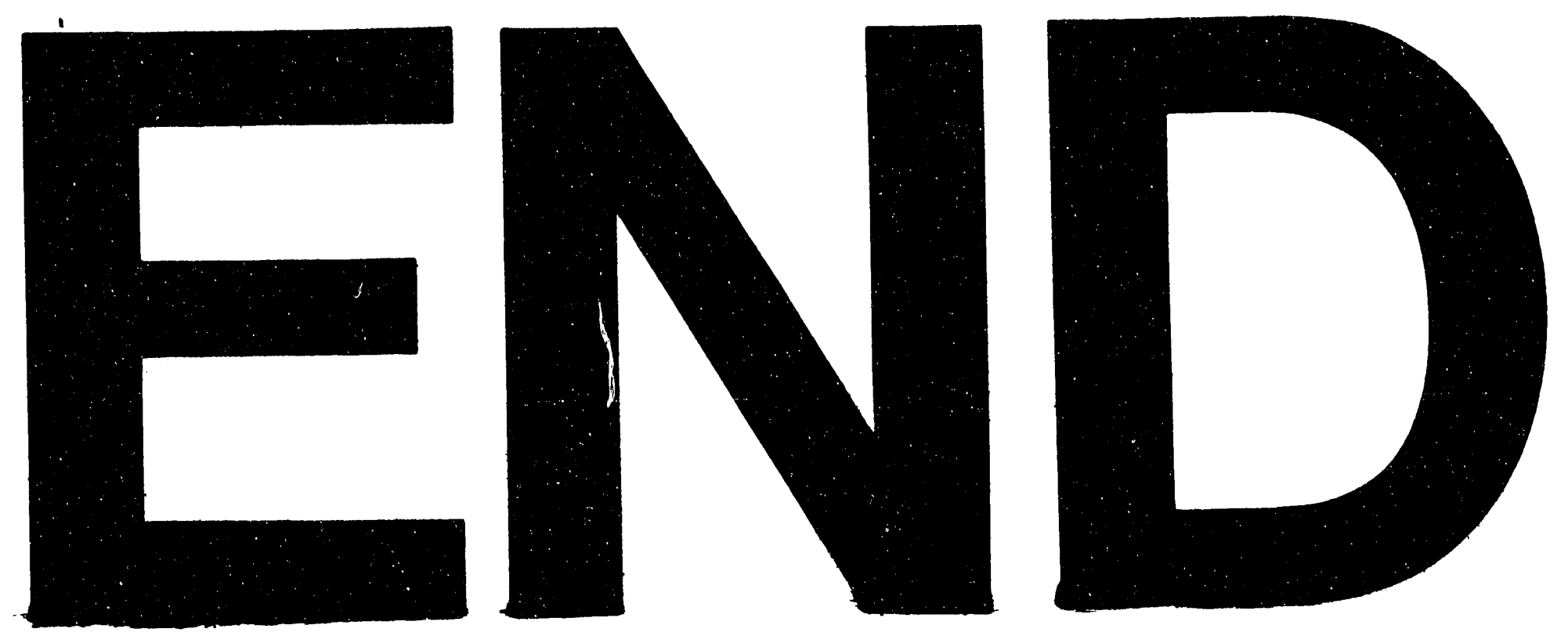

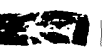
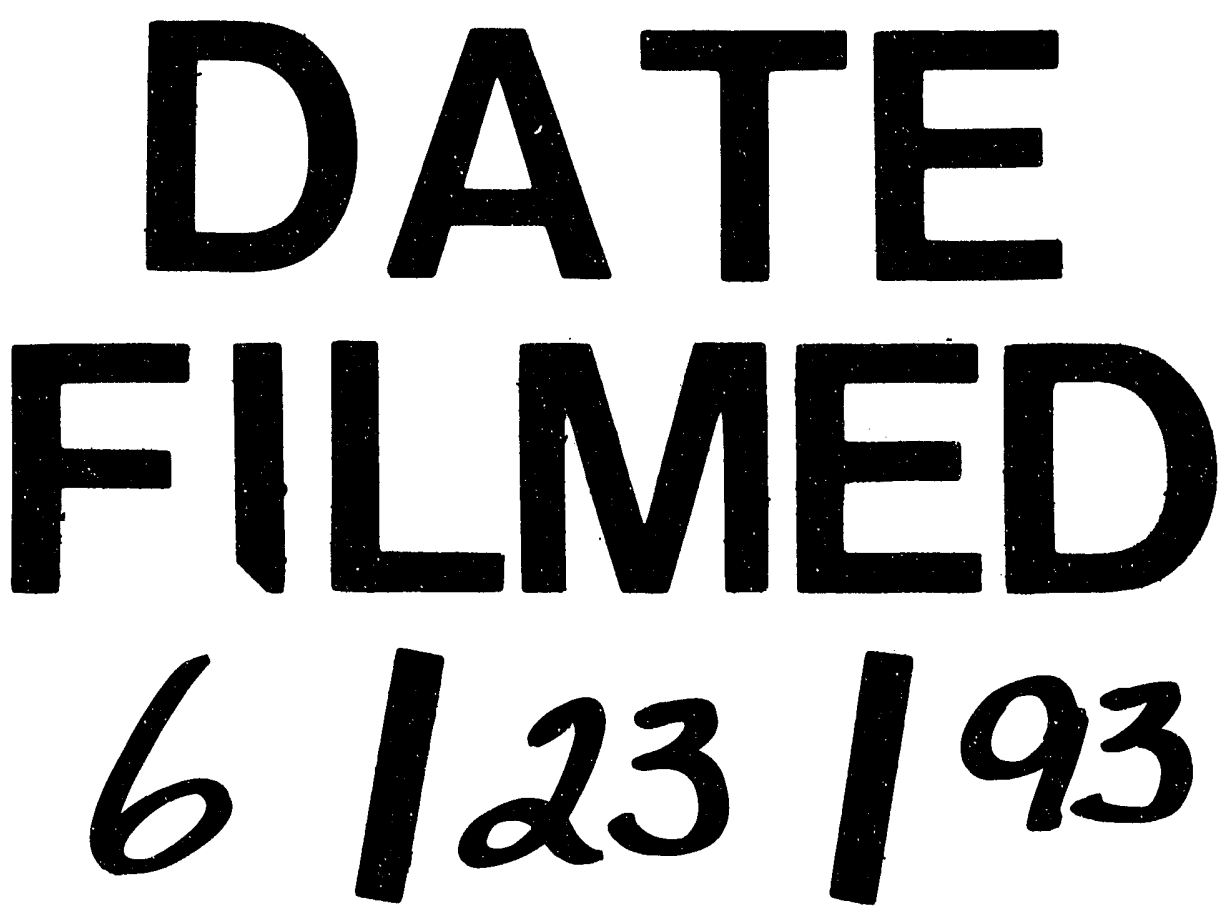
